\title{
Kähler metrics via Lorentzian Geometry in dimension four
}

https://doi.org/10.1515/coma-2020-0002

Received July 7, 2019; accepted October 15, 2019

\begin{abstract}
Given a semi-Riemannian 4-manifold $(M, g)$ with two distinguished vector fields satisfying properties determined by their shear, twist and various Lie bracket relations, a family of Kähler metrics $g_{K}$ is constructed, defined on an open set in $M$, which coincides with $M$ in many typical examples. Under certain conditions $g$ and $g_{K}$ share various properties, such as a Killing vector field or a vector field with a geodesic flow. In some cases the Kähler metrics are complete. The Ricci and scalar curvatures of $g_{\kappa}$ are computed under certain assumptions in terms of data associated to $g$. Many examples are described, including classical spacetimes in warped products, for instance de Sitter spacetime, as well as gravitational plane waves, metrics of Petrov type $D$ such as Kerr and NUT metrics, and metrics for which $g_{K}$ is an SKR metric. For the latter an inverse ansatz is described, constructing $g$ from the SKR metric.
\end{abstract}

Keywords: Kahler metric, Lorentzian metric, shear, twist, integrable complex structures, warped products, de Sitter spacetime, Kerr metric, gravitational waves, pp waves, Petrov type.

MSC: 53B30, 53B55, 53C55

\section{Introduction}

In one of his open problem collections, S. T. Yau concludes a problem with the question

\section{"Can one go from complete Kähler manifolds \\ back to physically interesting spacetimes?"}

([34, Problem 89]). This study makes a contribution mostly in the opposite direction, by constructing Kähler metrics from Lorentzian 4-manifolds equipped with associated data.

To be sure, Yau's question is written in the context of the effort to make sense of the transformation between metrics known as Wick rotation. In contrast, the construction method presented in this study, which applies in arbitrary signature, is different, and more involved, than Wick rotation. But it is invariantly defined, and its two variants can be carried out on a variety of classical spacetimes, such as de Sitter, Kerr and gravitational plane waves. For one class of Kähler metrics, which includes the extremal metric conformal to the Page metric, we give a kind of inverse construction: the Kähler metric is the input data for an ansatz producing Lorentzian 4-manifolds, for which the construction method recovers the Kähler metric on an open dense set.

In some cases the construction yields complete Kähler metrics, or ones that extend to a larger compact manifold. There are also curvature-distinguished Kähler metrics, such as Kähler-Einstein ones, that arise from this construction. Some of those are described in the sequel [2] to this work.

It is well-known that a Lorentzian metric is never compatible with any given almost complex structure. Thus relating notions of Lorentzian and complex geometry is not a straightforward process. Attempts to achieve this date back at least to the 1960's, and one of its most well-known outcomes is the invention

Amir Babak Aazami: Department of Mathematics and Computer Science, Clark University, Worcester, MA, E-mail: Aaazami@clarku.edu

*Corresponding Author: Gideon Maschler: Department of Mathematics and Computer Science, Clark University, Worcester, MA, E-mail: Gmaschler@clarku.edu 
of twistor theory. Connections relating Lorentzian geometry specifically to Kähler geometry have also been made, some focusing on analogous structures in the two geometries, partly based on considerations from spin geometry $[6,18,26,32]$. Our construction links the two geometries more directly, and is given in terms of standard differential geometric data, but is closely related to some of the papers just cited, and especially to [25]. Flaherty's classical manuscript [16] also attempts such a direct link, but ultimately follows a different path.

In more detail, given an oriented four-manifold with a semi-Riemannian metric $g$ and two distinguished vector fields $\boldsymbol{k}, \boldsymbol{t}$ satisfying certain "admissibility" conditions (Definition 4.1), we construct an integrable almost complex structure and a family of exact symplectic forms. Fixing one such form, it will be compatible with this complex structure and thus yield a Kähler metric $g_{K}$ on some open set (see Theorem 3 and Proposition 10.1). In favorable cases, which include almost all of our examples, this open set coincides with the whole manifold. Such a Kähler metric is not always complete, but we do give complete examples (see subsection 8.5).

In the case $g$ is Lorentzian and $\boldsymbol{k}$ is null with a geodesic flow, such symplectic forms were defined by the first author in [1], which constituted the original motivation for this study.

Nondegeneracy of the symplectic form, and integrability of the almost complex structure are related to invariants associated with $\boldsymbol{k}$, namely its twist and shear, respectively, first introduced in General Relativity [29]. We employ variants of these notions defined relative to a direct sum decomposition of the tangent bundle.

The shear-free condition for a single vector field and its relation to integrability of almost complex structures was studied in $[8,9]$. But our shear condition is more general, as we show in an example (see Section 11). In the shear-free case, our Kähler metrics are ambihermitian and at times ambiKähler, in the sense of [5].

We actually give two theorems related to integrability. A Newman-Penrose version of the conditions of the second (Theorem 2) is given in Formula (VIII.5) of [16], suitable for the special case where $\boldsymbol{k}, \boldsymbol{t}$ are part of a null tetrad (a special type of frame). In some of our examples the frame is not of this type (see also Remark 3.6).

Ricci curvature computations of some of these Kähler metrics appear mostly in [2], but also in Section 6 under certain assumptions.

Lorentzian metrics inducing Kähler metrics which are SKR are given in Section 7. The latter were first introduced in [13], in the context of the classification of conformally-Einstein Kähler metrics (Section 7).

In Section 8 we show that a large class of Lorentzian warped products are admissible. The same is shown in Section 9 for gravitational plane waves.

For metrics of Petrov type $D$, we give in Section 10 three examples in which the theory is implemented and produces Kähler metrics: the Kerr metric, a class of NUT metrics and a metric conformal to the Kerr metric. The first two of these examples require a different variant of the construction of an associated Kähler metric. The complex structure for the NUT metric was first described in [16]. Dixon [15] has recently studied another Kähler metric Wick-rotated from the Kerr metric on a domain in Kerr spacetime, and showed it is ambitoric (see also [4]).

\section{Shear and twist}

In this preliminary section we introduce variants of the notions of shear and twist, the optical invariants that will have a significant role in what follows. After describing their expressions in appropriate frames, we compare our version to the more standard one for null vector fields with a geodesic or pre-geodesic flow. We then describe a few known applications valid especially for 3-manifolds, which will be needed in Section 8 . 


\subsection{Relative versions of shear and twist}

Our notions of shear and twist will differ somewhat from their common usage in the Physics literature, and also from mathematical references such as [9]. The need for these atypical definitions arises, in small part, from their application to vector fields on semi-Riemannian manifolds which may not be null, or even of constant length. But more importantly, the difference is attributed to the fact that we consider two (pointwise linearly independent) distinguished vector fields, rather than just one. Thus our shear and twist will be defined not with respect to the orthogonal complement of a single vector field, but relative to a decomposition of the tangent bundle into an orthogonal direct sum of distributions, one of which is spanned by these two vector fields. Specifically, for a semi-Riemannian manifold $M$, let

$$
T M=\mathcal{V} \oplus \mathcal{H}
$$

be an orthogonal decomposition of the tangent bundle into two (necessarily nondegenerate) distributions $\mathcal{V}, \mathcal{H}$. Let $\pi: T M \rightarrow \mathcal{H}$ be the projection relative to this decomposition, and let $\nabla$ denote the Levi-Civita connection of $M$.

Definition 2.1. Let $X$ be a nowhere-vanishing vector field taking values in $\mathcal{V}$. The relative shear operator and relative twist operator of $X$ are defined, respectively, as the $\mathcal{H} \rightarrow \mathcal{H}$ operators given by

$$
\begin{aligned}
& \text { relative shear: } \nabla^{o} X:=\text { trace-free symmetric part of }\left.\pi \circ \nabla X\right|_{\mathcal{H}}, \\
& \text { relative twist: } \nabla^{S} X:=\text { skew-symmetric part of }\left.\pi \circ \nabla X\right|_{\mathcal{H}},
\end{aligned}
$$

where $\nabla X$ refers to the linear operator $v \mapsto \nabla_{v} X$ on the tangent bundle. If $\nabla^{\circ} X$ or $\nabla^{s} X$ vanishes, then $X$ is shearfree or twist-free, respectively.

These relative optical invariants will be applied throughout most of the paper, and we will often omit the term "relative" when using them.

In all our applications the rank of $\mathcal{H}$ will be two. In this case the following related entity is real-valued, and will play an important role.

Definition 2.2. The (relative) twist function of $X$ is

$$
|\iota|=\left|\iota^{X}\right|:=2 \sqrt{\operatorname{det}\left(\nabla^{s} X\right)} .
$$

If $\iota^{X}$ is nowhere vanishing, then the flow of $X$ is called everywhere twisting.

The reason for the notation $|\iota|$ is that the twist function is the absolute value of a function $\iota$ defined in the next subsection with respect to an orthonormal frame of $\mathcal{H}$.

In our most common application, the manifold will be Lorentzian of dimension four, and admit an almost complex structure. Then $\mathcal{V}$ will be the complex span of some vector field $X$. In this Lorentzian setting, we will always choose $\mathcal{V}$ to be timelike, so that $\mathcal{H}=\mathcal{V}^{\perp}$ will be spacelike, i.e. $\left.g\right|_{\mathcal{H}}$ will be positive definite.

\subsection{Frame representation}

In the setting of the previous subsection, assume $\mathcal{H}$ has rank two, and let $\boldsymbol{x}, \boldsymbol{y}$ be an ordered orthonormal frame for $\mathcal{H}$. Then at each point, the matrix of $\left.\pi \circ \nabla X\right|_{\mathcal{H}}$ (like that of $\left.\nabla X\right|_{\mathcal{H}}$ ) with respect to $\{\boldsymbol{x}, \boldsymbol{y}\}$ is given by

$$
\left[\left.\pi \circ \nabla X\right|_{\mathcal{H}}\right]_{\boldsymbol{x}, \boldsymbol{y}}=\left[\begin{array}{ll}
g\left(\nabla_{\boldsymbol{x}} X, \boldsymbol{x}\right) & g\left(\nabla_{\boldsymbol{y}} X, \boldsymbol{x}\right) \\
g\left(\nabla_{\boldsymbol{x}} X, \boldsymbol{y}\right) & g\left(\nabla_{\boldsymbol{y}} X, \boldsymbol{y}\right)
\end{array}\right] .
$$

Thus the shear operator of $X$ is

$$
\left[\nabla^{o} X\right]_{\boldsymbol{x}, \boldsymbol{y}}=\left[\begin{array}{cc}
-\sigma_{1} & \sigma_{2} \\
\sigma_{2} & \sigma_{1}
\end{array}\right]
$$


where the entries are the shear coefficients

$$
\begin{aligned}
& \sigma_{1}:=\frac{1}{2}\left[g\left(\nabla_{\boldsymbol{y}} X, \boldsymbol{y}\right)-g\left(\nabla_{\boldsymbol{x}} X, \boldsymbol{x}\right)\right]=\frac{1}{2}[g([X, \boldsymbol{x}], \boldsymbol{x})-g([X, \boldsymbol{y}], \boldsymbol{y})], \\
& \sigma_{2}:=\frac{1}{2}\left[g\left(\nabla_{\boldsymbol{y}} X, \boldsymbol{x}\right)+g\left(\nabla_{\boldsymbol{x}} X, \boldsymbol{y}\right)\right]=-\frac{1}{2}[g([X, \boldsymbol{x}], \boldsymbol{y})+g([X, \boldsymbol{y}], \boldsymbol{x})] .
\end{aligned}
$$

While these coefficients are frame-dependent, $\sigma_{1}^{2}+\sigma_{2}^{2}=-\operatorname{det} \nabla^{o} X$ is an invariant quantity.

The twist operator of $X$ is given in this frame by

$$
\left[\nabla^{S} X\right]_{x, y}=\left[\begin{array}{cc}
0 & \iota^{X} / 2 \\
-\iota^{X} / 2 & 0
\end{array}\right],
$$

where

$$
\iota^{X}=\iota:=g\left(\nabla_{\boldsymbol{y}} X, \boldsymbol{x}\right)-g\left(\nabla_{\boldsymbol{x}} X, \boldsymbol{y}\right)=g(X,[\boldsymbol{x}, \boldsymbol{y}]) .
$$

As with the shear coefficients, $\left(l^{X}\right)^{2}=4 \operatorname{det} \nabla^{s} X$ is invariant, namely it is the square of the twist function.

Remark 2.3. Starting from Section 3 and throughout the paper, the distribution $\mathcal{H}$ will always be oriented. We will use this to fix the sign of $\iota$ by the convention that $\iota$ is always computed as in (4) with respect to an oriented orthonormal frame, oriented to agree with the orientation of $\mathcal{H}$.

Note that relations (3) have interesting consequences. For example, if $X$ is a vertical field for a Riemannian submersion, while $\mathcal{H}$ is its horizontal distribution, then $X$ is shear-free, because the bracket of a vertical vector field with a horizontal one is vertical.

\subsection{Classical shear and twist}

We briefly compare here our versions of relative twist and relative shear with more standard notions (see e.g., [31] and [28, Chapter 5]). These standard notions will generally not be used further, except for a version which applies to Riemannian 3-manifolds, see below.

First, for later purposes we give some terminology related to the equation

$$
\nabla_{\boldsymbol{k}} \boldsymbol{k}=\alpha \boldsymbol{k} \text { for some smooth function } \alpha \text { on } M .
$$

Remark 2.4. If equation (5) holds, $\boldsymbol{k}$ will be called pre-geodesic; if $\alpha$ is not identically zero it will be called strictly pre-geodesic, and geodesic if $\nabla_{\boldsymbol{k}} \boldsymbol{k}=0$.

If $\boldsymbol{k}$ is a null pre-geodesic field on a Lorentzian 4-manifold, its covariant derivative induces an operator $D: \boldsymbol{k}^{\perp} / \boldsymbol{k} \longrightarrow \boldsymbol{k}^{\perp} / \boldsymbol{k}$ on a quotient of its orthogonal space. Then the (non-relative) shear and twist of $\boldsymbol{k}$ are defined as the trace-free symmetric, respectively antisymmetric components of this operator.

For the relative case, $\mathcal{H}$ in Definition 2.1 is the image of a chosen embedding $\boldsymbol{k}^{\perp} / \boldsymbol{k} \rightarrow \boldsymbol{k}^{\perp}$, and the definition depends, of course, on this choice.

Let $(N, g)$ be a Riemannian 3-manifold, $\boldsymbol{k}$ a vector field on $N$ of unit length, so that $\boldsymbol{k} \oplus \boldsymbol{k}^{\perp}=T N$. The relevant bundle map is now $D: \boldsymbol{k}^{\perp} \longrightarrow \boldsymbol{k}^{\perp}, D(v):=\nabla_{v} \boldsymbol{k}$, which is well defined as $\boldsymbol{k}$ has constant length ${ }^{1}$. In terms of an orthonormal frame $\{\boldsymbol{x}, \boldsymbol{y}\}$ of $\boldsymbol{k}^{\perp}$, the shear and twist of $\boldsymbol{k}$ are still given by formulas (3) and (4).

\subsection{Preliminary applications in dimensions 3 and 4}

For a Riemannian 3-manifold $(N, g)$ with $\boldsymbol{k}, \boldsymbol{x}, \boldsymbol{y}$ as in the previous section, Frobenius' theorem implies that $\iota^{k}$, given as in (4)

$$
\iota^{k}=g(k,[x, y])
$$

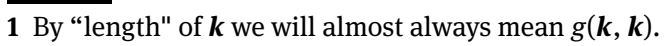


vanishes identically if and only if the orthogonal complement $\boldsymbol{k}^{\perp}$ of $\boldsymbol{k}$ is integrable. We will be interested in the diametrically opposed situation where the frame independent twist function $|\iota|$ is nowhere vanishing, so that $\boldsymbol{k}^{\perp}$ is nowhere integrable: at any $p \in M$, there is no embedded submanifold $S$ containing $p$ such that $T_{q} S=\boldsymbol{k}_{q}^{\perp}$ for all $q \in S$. We record this analysis together with a related result, proven in [20].

Lemma 2.5. If a unit length vector field $\boldsymbol{k}$ on a Riemannian 3-manifold is complete, has geodesic flow, and $\operatorname{Ric}(\boldsymbol{k}, \boldsymbol{k})>0$, then $\boldsymbol{k}^{\perp}$ is nowhere integrable. The latter occurs if and only if $\boldsymbol{k}$ is everywhere twisting.

Next, we record the following well-known lemma, omitting its standard proof.

Lemma 2.6. A unit length vector field on a Riemannian 3-manifold is a Killing vector field if and only if it is geodesic, divergence-free, and shear-free.

The last two lemmas will only be applied in Section 8 .

Similarly, On a Lorentzian 4-manifold the integrability of $\boldsymbol{k}^{\perp}$, for a null pre-geodesic vector field $\boldsymbol{k}$, is completely determined by the vanishing of the twist function $|\boldsymbol{\iota}|=|g(\boldsymbol{k},[\boldsymbol{x}, \boldsymbol{y}])|($ see $[28, \mathrm{Ch} .5])$. Moreover, a wellknown result holds in analogy with Lemma 2.5; see, e.g. [1] for a proof.

Lemma 2.7. If a null vector field $\boldsymbol{k}$ with geodesic flow on a Lorentzian 4-manifold is complete and $\operatorname{Ric}(\boldsymbol{k}, \boldsymbol{k})>0$, then $\boldsymbol{k}^{\perp}$ is nowhere integrable. The latter occurs if and only if $\boldsymbol{k}$ is everywhere twisting.

\section{Almost complex structures and integrability}

In this section we introduce a key component of this work, namely an almost complex structure attached to a semi-Riemannian 4-manifold equipped with certain data involving two vector fields. We investigate its integrability and related properties.

\subsection{Admissible almost complex structures}

Let $(M, g)$ be an oriented semi-Riemannian 4-manifold, with two vector fields $\boldsymbol{k}_{+}, \boldsymbol{k}_{-}$. Let $\mathcal{V}:=\operatorname{span}\left(\boldsymbol{k}_{+}, \boldsymbol{k}_{-}\right)$ denote the distribution spanned at each point $p$ by $\boldsymbol{k}_{+}\left|p, \boldsymbol{k}_{-}\right| p$. We assume that

$$
\boldsymbol{k}_{+}, \boldsymbol{k}_{-} \text {are everywhere linearly independent, }
$$

and

$$
\mathcal{H}:=\operatorname{span}\left(\boldsymbol{k}_{+}, \boldsymbol{k}_{-}\right)^{\perp} \text { is spacelike. }
$$

Remark 3.1. Note in particular that $\boldsymbol{k}_{ \pm}$then have no zeros. Condition (7) means that $\left.g\right|_{\mathcal{H}}$ is positive definite at each point, in particular it is pointwise nondegenerate, which also implies that $\left.g\right|_{\mathcal{V}}$ is pointwise nondegenerate. This last condition together with (6) are equivalent to

$$
A:=\left[\begin{array}{ll}
g\left(\boldsymbol{k}_{+}, \boldsymbol{k}_{+}\right) & g\left(\boldsymbol{k}_{+}, \boldsymbol{k}_{-}\right) \\
g\left(\boldsymbol{k}_{-}, \boldsymbol{k}_{+}\right) & g\left(\boldsymbol{k}_{-}, \boldsymbol{k}_{-}\right)
\end{array}\right] \text {is everywhere nonsingular, }
$$

Thus (6) and (7) are equivalent to (8) and (7). Finally, if $g$ is Lorentzian, (7) is equivalent to

$$
\mathcal{V}=\operatorname{span}\left(\boldsymbol{k}_{+}, \boldsymbol{k}_{-}\right) \text {is timelike, }
$$

i.e. $\left.g\right|_{\mathcal{V}}$ has Lorentzian signature at each point. In that case (9) implies

$$
G:=\operatorname{det}(A)<0
$$


We consider an almost complex structure $J=J_{g, \boldsymbol{k}_{+}}$on $M$ defined as follows. First, we set $J \boldsymbol{k}_{+}:=\boldsymbol{k}_{-}, J \boldsymbol{k}_{-}:=-\boldsymbol{k}_{+}$ and extend these relations linearly on $\mathcal{V}$. Second, we note that the restriction of $g$ to $\mathcal{H}$ is positive definite, and $\mathcal{H}$ inherits an orientation because $M$ is oriented and the ordered pair $\boldsymbol{k}_{+}, \boldsymbol{k}_{-}$induces an orientation on $\mathcal{V}$. We thus define $\left.J\right|_{\mathcal{H}}$ to be the unique endmorphism of $\mathcal{H}$ whose square is minus the identity, which is additionally an isometry of $\left.g\right|_{\mathcal{H}}$ and respects the orientation on $\mathcal{H}$. Finally, we define $J$ by extending $\left.J\right|_{\mathcal{V}},\left.J\right|_{\mathcal{H}}$ linearly on $T M=\mathcal{V} \oplus \mathcal{H}$.

Definition 3.2. An almost complex structure $J=J_{g, \boldsymbol{k}_{ \pm}}$on an oriented 4-manifold is called admissible if it is constructed as above, using a semi-Riemannian metric $g$, and two vector fields $\boldsymbol{k}_{ \pm}$satisfying (6), (7). If the integrability relations (11) below also hold, $J$ will be called an admissible complex structure.

\subsection{Integrability}

Integrability of an almost complex structure $J$ implies the manifold admits complex coordinates. It is defined by the vanishing of the Nijenhuis tensor

$$
N(a, b)=[J a, J b]-J[J a, b]-J[a, J b]-[a, b] .
$$

We now give sufficient conditions for integrability of an admissible almost complex structure.

Theorem 1. An admissible almost complex structure $J=J_{g, \boldsymbol{k}_{ \pm}}$is integrable if the following three conditions hold:

$$
\text { i) }\left[\boldsymbol{k}_{ \pm}, \Gamma(\mathcal{H})\right] \subset \Gamma(\mathcal{H}), \quad \text { ii) } J \nabla^{o} \boldsymbol{k}_{+}=\nabla^{o} J \boldsymbol{k}_{+} \text {on } \mathcal{H} .
$$

The notation of i) means that the Lie bracket operation with $\boldsymbol{k}_{+}$or $\boldsymbol{k}_{-}$sends any vector field in $\mathcal{H}$ to another such vector field. The shear notation in ii) is as in Definition 2.1.

Note that condition ii) is invariant under multiplying both vector fields $\boldsymbol{k}_{ \pm}$by a common factor, while conditions i) are not. This has the consequence that the conclusion of the theorem still holds if conditions i) are replaced by the requirement that they hold instead with $\boldsymbol{k}_{ \pm}$replaced by some common nowhere vanishing multiple of themselves.

Proof. We examine the Nijenhuis tensor of $J$ for a frame $\left\{\boldsymbol{k}_{ \pm}, \boldsymbol{x}_{ \pm}\right\}$, where $\left\{\boldsymbol{x}_{+}, \boldsymbol{x}_{-}=J \boldsymbol{x}_{+}\right\}$is an oriented orthonormal frame for $\left.g\right|_{\mathcal{H}}$. Clearly $N$ vanishes on any pair $a$, $J a$. The relation $N(a, b)=J N(a, J b)$ along with the antisymmetry of $N(a, b)$ imply that it is enough to check the vanishing for the pair $\boldsymbol{k}_{+}, \boldsymbol{x}_{+}$.

We thus analyze

$$
\begin{aligned}
N\left(\boldsymbol{k}_{+}, \boldsymbol{x}_{+}\right) & =\left[J \boldsymbol{k}_{+}, J \boldsymbol{x}_{+}\right]-J\left[J \boldsymbol{k}_{+}, \boldsymbol{x}_{+}\right]-J\left[\boldsymbol{k}_{+}, J \boldsymbol{x}_{+}\right]-\left[\boldsymbol{k}_{+}, \boldsymbol{x}_{+}\right] \\
& =\left[J \boldsymbol{k}_{+}, \boldsymbol{x}_{-}\right]-J\left[J \boldsymbol{k}_{+}, \boldsymbol{x}_{+}\right]-J\left[\boldsymbol{k}_{+}, \boldsymbol{x}_{-}\right]-\left[\boldsymbol{k}_{+}, \boldsymbol{x}_{+}\right] .
\end{aligned}
$$

Conditions (11)i) along with the $J$-invariance of $\mathcal{H}$ imply via (12) that $N\left(\boldsymbol{k}_{+}, \boldsymbol{x}_{+}\right)$is a section of $\mathcal{H}$.

Next, taking the inner product of the right-hand side of (12) with $\boldsymbol{x}_{+}$, while employing the fact that $\left.g\right|_{\mathcal{H}}$ is hermitian, we arrive at the following expression:

$$
\begin{aligned}
g\left(N\left(\boldsymbol{k}_{+}, \boldsymbol{x}_{+}\right), \boldsymbol{x}_{+}\right) & =g\left(\left[J \boldsymbol{k}_{+}, \boldsymbol{x}_{-}\right], \boldsymbol{x}_{+}\right)+g\left(\left[J \boldsymbol{k}_{+}, \boldsymbol{x}_{+}\right], \boldsymbol{x}_{-}\right) \\
& +g\left(\left[\boldsymbol{k}_{+}, \boldsymbol{x}_{-}\right], \boldsymbol{x}_{-}\right)-g\left(\left[\boldsymbol{k}_{+}, \boldsymbol{x}_{+}\right], \boldsymbol{x}_{+}\right)
\end{aligned}
$$

Referring now to the shear coefficient expressions (3), the above yields the following two equalities, the second obtained in analogy with the first:

$$
\begin{aligned}
& g\left(N\left(\boldsymbol{k}_{+}, \boldsymbol{x}_{+}\right), \boldsymbol{x}_{+}\right)=-2 \sigma_{1}^{\boldsymbol{k}_{+}}-2 \sigma_{2}^{j \boldsymbol{k}_{+},}, \\
& g\left(N\left(\boldsymbol{k}_{+}, \boldsymbol{x}_{+}\right), \boldsymbol{x}_{-}\right)=+2 \sigma_{2}^{\boldsymbol{k}_{+}}-2 \sigma_{1}^{j \boldsymbol{k}_{+},},
\end{aligned}
$$

where the shear-related notations are as in Section 2.2. Since the action of the shear matrix (2) on each of the standard basis vectors in $\mathbb{R}^{2}$ yields $\left(-\sigma_{1}, \sigma_{2}\right)$ and $\left(\sigma_{2}, \sigma_{1}\right)$, respectively, the last two equations yield the invariant formula

$$
\iota_{\boldsymbol{k}_{+}} N=2\left(\nabla^{o} \boldsymbol{k}_{+}-\nabla^{o} J \boldsymbol{k}_{+} \circ J\right) \text { on } \mathcal{H} .
$$


In fact, they yield equality of both sides on $\boldsymbol{x}_{+}$, and we obtain it on $\boldsymbol{x}_{-}$because of both $N\left(\boldsymbol{k}_{+}, \boldsymbol{x}_{-}\right)=-J N\left(\boldsymbol{k}_{+}, \boldsymbol{x}_{+}\right)$ and the fact that $\left.J\right|_{\mathcal{H}}$ anticommutes with any trace-free symmetric operator $P$ acting on $\mathcal{H}$. This last fact holds since $J$ makes $\left.g\right|_{\mathcal{H}_{C}}$ hermitian, so that the adjoint of $P J$ is $-J P$, while the trace-free condition implies $g\left(P J \boldsymbol{x}_{+}, \boldsymbol{x}_{-}\right)=g\left(P \boldsymbol{x}_{-}, \boldsymbol{x}_{-}\right)=-g\left(P \boldsymbol{x}_{+}, \boldsymbol{x}_{+}\right)=g\left(P J \boldsymbol{x}_{-}, \boldsymbol{x}_{+}\right)$, so that $P J$ is also self-adjoint.

By applying the last mentioned fact to (13), the theorem follows.

A generalization of this theorem will appear in subsection 3.4.

Remark 3.3. It is easily seen that if $J$ satisfies the conditions of this theorem, the almost complex structure defined just as $J$, but with respect to the opposite orientation, will also be integrable if $\boldsymbol{k}_{ \pm}$are shear-free.

Note that (8) implies that (11)i) is equivalent to the four conditions

$$
g\left(\left[\boldsymbol{k}_{ \pm}, \cdot\right], \boldsymbol{k}_{ \pm}\right)=0 \text { and } g\left(\left[\boldsymbol{k}_{ \pm}, \cdot\right], \boldsymbol{k}_{\mp}\right)=0 \text { on } \mathcal{H} .
$$

The converse of Theorem 1 does not hold in general. However, we have

Proposition 3.4. If three of the conditions (14) hold, and $N=0$, then the fourth, along with ii) of (11), also hold.

Proof. Assume $N=0$ and, for example, $g\left(\left[\boldsymbol{k}_{+}, \cdot\right], \boldsymbol{k}_{+}\right)=g\left(\left[\boldsymbol{k}_{-}, \cdot\right], \boldsymbol{k}_{-}\right)=g\left(\left[\boldsymbol{k}_{+}, \cdot\right], \boldsymbol{k}_{-}\right)=0$ on $\mathcal{H}$. Let

$$
A=\left[g\left(\boldsymbol{k}_{ \pm}, \boldsymbol{k}_{ \pm}\right)\right]:=\left[\begin{array}{ll}
p & r \\
r & q
\end{array}\right],
$$

and express the following vector fields in our standard frame:

$\left[\boldsymbol{k}_{-}, \boldsymbol{x}_{-}\right]=a \boldsymbol{k}_{+}+b \boldsymbol{k}_{-}+\cdots,\left[\boldsymbol{k}_{-}, \boldsymbol{x}_{+}\right]=c \boldsymbol{k}_{+}+d \boldsymbol{k}_{-}+\cdots$, for coefficients $a, b, c, d$. The coefficients $a, b$ may be obtained by applying the inverse of $A$ to the vector $\left(g\left(\left[\boldsymbol{k}_{-}, \boldsymbol{x}_{-}\right], \boldsymbol{k}_{+}\right), g\left(\left[\boldsymbol{k}_{-}, \boldsymbol{x}_{-}\right], \boldsymbol{k}_{-}\right)\right)=\left(g\left(\left[\boldsymbol{k}_{-}, \boldsymbol{x}_{-}\right], \boldsymbol{k}_{+}\right), 0\right)$, while for obtaining $c, d$ one applies the same matrix to $\left(g\left(\left[\boldsymbol{k}_{-}, \boldsymbol{x}_{+}\right], \boldsymbol{k}_{+}\right), g\left(\left[\boldsymbol{k}_{-}, \boldsymbol{x}_{+}\right], \boldsymbol{k}_{-}\right)\right)=\left(g\left(\left[\boldsymbol{k}_{-}, \boldsymbol{x}_{+}\right], \boldsymbol{k}_{+}\right), 0\right)$. On the other hand similar coefficients for $\left[\boldsymbol{k}_{+}, \boldsymbol{x}_{ \pm}\right]$all vanish by our assumptions. Substituting the above expressions for the Lie bracket terms in $N$, we see that

$$
N\left(\boldsymbol{k}_{+}, \boldsymbol{x}_{+}\right)=(a+d) \boldsymbol{k}_{+}+(b-c) \boldsymbol{k}_{-}+\text {terms in } \mathcal{H} .
$$

As $N=0$, these coefficients of $\boldsymbol{k}_{ \pm}$vanish, and together with the above method of obtaining $a, b, c, d$ this gives the two equations $q B=r C,-r B=q C$, where $B=g\left(\left[\boldsymbol{k}_{-}, \boldsymbol{x}_{-}\right], \boldsymbol{k}_{+}\right), C=g\left(\left[\boldsymbol{k}_{-}, \boldsymbol{x}_{+}\right], \boldsymbol{k}_{+}\right)$. These equations in turn imply $\operatorname{qr}\left(B^{2}+C^{2}\right)=0$, which easily leads to $B=C=0$, since $q$ and $r$ cannot both vanish. We thus see that $g\left(\left[\boldsymbol{k}_{-}, \cdot\right], \boldsymbol{k}_{+}\right)$vanishes on $\mathcal{H}$. Then (ii) of (11) follows as in Theorem 1.

\subsection{Geometric conditions implying (14)}

We now consider the question of whether there are geometric circumstances in which any one of the four conditions (14) holds automatically. All of them, of course, must be satisfied simultaneously for condition (11)i) to hold. The observations we note here, for which we omit the straightforward proofs, will serve to verify integrability of admissible almost complex structures appearing in our examples in Sections 8-11.

Remark 3.5. For $\boldsymbol{k}=\boldsymbol{k}_{ \pm}$, relation $g([\boldsymbol{k}, \cdot], \boldsymbol{k})=0$ holds on $\mathcal{H}$ in two cases:

a) $\boldsymbol{k}$ is a pre-geodesic vector field of constant length, or

b) $\boldsymbol{k}$ is Killing.

Next, condition $g\left(\left[\boldsymbol{k}_{+}, \cdot\right], \boldsymbol{k}_{-}\right)=0$ holds on $\mathcal{H}$ if the following two conditions both hold:

$$
\begin{aligned}
& \text { i) } \boldsymbol{k}_{-}=\ell \nabla \tau \text {, where } \nabla \ell \in \Gamma(\mathcal{V}) \text {, and } \\
& \text { ii) } \nabla\left(g\left(\boldsymbol{k}_{+}, \boldsymbol{k}_{-}\right)\right) \in \Gamma(\mathcal{V}) \text {. }
\end{aligned}
$$


Here $\tau, \ell$ are smooth functions. Note that this will also follow if i) of (16) is replaced by $g\left(\boldsymbol{k}_{+}, \boldsymbol{k}_{-}\right)=0$.

If (16)i) holds, the condition $g\left(\left[\boldsymbol{k}_{-}, \cdot\right], \boldsymbol{k}_{+}\right)=0$ on $\mathcal{H}$ can be translated into the form

$$
g\left(\nabla_{\boldsymbol{k}_{+}} \boldsymbol{k}_{-}+\nabla_{\boldsymbol{k}_{-}} \boldsymbol{k}_{+}, \cdot\right)=0 \text { on } \mathcal{H} .
$$

When both $\boldsymbol{k}_{+}, \boldsymbol{k}_{-}$are pre-geodesic, and $\mathcal{H}$ is integrable, condition (17) guarantees that $\mathcal{V}$ is the horizontal distribution for a semi-Riemannian submersion. But integrability of $\mathcal{H}$ will never, in fact, occur in the circumstances we will be considering later.

\subsection{Integrability for split-adjoint admissible almost complex structures}

An admissible almost complex structure $J=J_{g, \boldsymbol{k}_{ \pm}}$will be called split-adjoint if $\left.J\right|_{\mathcal{V}}$ is $\left.g\right|_{\mathcal{V}}$-self-adjoint. We now give a necessary and sufficient condition for the integrability of such $J$.

Theorem 2. Let $J=J_{g, \boldsymbol{k}_{ \pm}}$be a split-adjoint admissible almost complex structure. Then $J$ is integrable if and only if the following conditions hold:

$$
\begin{aligned}
& \text { i) } g\left(\left[\boldsymbol{k}_{-}, J \boldsymbol{x}\right], \boldsymbol{k}_{+}\right)-g\left(\left[\boldsymbol{k}_{+}, J \boldsymbol{x}\right], \boldsymbol{k}_{-}\right)-g\left(\left[\boldsymbol{k}_{+}, \boldsymbol{x}\right], \boldsymbol{k}_{+}\right)-g\left(\left[\boldsymbol{k}_{-}, \boldsymbol{x}\right], \boldsymbol{k}_{-}\right)=0 \text {, } \\
& \text { ii) } J \nabla^{o} \boldsymbol{k}_{+}=\nabla^{o} J \boldsymbol{k}_{+} \text {on } \mathcal{H},
\end{aligned}
$$

where $\boldsymbol{x}$ in $i$ ) is any vector field lying in $\mathcal{H}$.

Proof. Recall from Theorem 1 that it is enough to analyze the vanishing of $N\left(\boldsymbol{k}_{+}, \boldsymbol{x}_{+}\right)=\left[\boldsymbol{k}_{-}, J \boldsymbol{x}_{+}\right]-J\left[\boldsymbol{k}_{+}, J \boldsymbol{x}_{+}\right]-$ $\left[\boldsymbol{k}_{+}, \boldsymbol{x}_{+}\right]-J\left[\boldsymbol{k}_{-}, \boldsymbol{x}_{+}\right]$, where $\boldsymbol{x}_{+}, \boldsymbol{x}_{-}=J \boldsymbol{x}_{+}$is a local oriented orthonormal frame for $\mathcal{H}$. Taking the inner product of this expression with $\boldsymbol{x}_{ \pm}$has been carried out in Theorem 1, and led to the shear-condition. This remains unchanged. Condition i) is obtained by first taking the inner product of $N\left(\boldsymbol{k}_{+}, \boldsymbol{x}_{+}\right)$with $\boldsymbol{k}_{ \pm}$, and then employing the self-adjointness of $\left.J\right|_{\mathcal{V}}$, to shift $J$ in the middle two terms in $N\left(\boldsymbol{k}_{+}, \boldsymbol{x}_{+}\right)$from the Lie bracket to the other vector field in the metric expression. Note finally that condition i) is tensorial on $\mathcal{H}$.

Remark 3.6. $\left.J\right|_{\mathcal{V}}$ is self-adjoint if and only if $\boldsymbol{k}_{ \pm}$have lengths of opposite signs. This makes Theorem 2 significant in two important cases which appear in our examples: if both $\boldsymbol{k}_{ \pm}$are null, or if they have nonzero lengths of opposite signs. Flaherty [16], already mentioned in the introduction, has given a Newman-Penrose version of Theorem 2 for a special case of the first case, namely if $\boldsymbol{k}_{ \pm}$are part of a null tetrad. Examples of the first case appear in Sections 9 and 10, while those of the second case are in Sections 7 and 11, in which $\boldsymbol{k}_{+}, \boldsymbol{k}_{-}$are additionally orthogonal.

In the following corollary $M, g, \boldsymbol{k}_{ \pm}, \mathcal{V}, \mathcal{H}$ are as in subsection 3.1 with (6), (7) holding, and we only list additional assumptions.

Corollary 3.7. Let $\boldsymbol{k}_{ \pm}$be null shear-free vector fields and $f_{1}, f_{2}$ nowhere vanishing smooth functions on $M$. Assume $J:=J_{g, \boldsymbol{k}_{ \pm}}$is an admissible complex structure. Then the admissible almost complex structure $\tilde{J}:=J_{g, f_{1} \boldsymbol{k}_{+}, f_{2} \boldsymbol{k}_{-}}$ is integrable if and only if $\nabla\left(f_{1} / f_{2}\right) \in \Gamma(\mathcal{V})$.

We omit the proof. Note that $\tilde{J}$ is indeed an admissible almost complex structure, since replacing $\boldsymbol{k}_{ \pm}$by $f_{1} \boldsymbol{k}_{+}$,

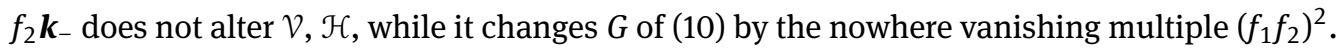

\subsection{Commuting $\boldsymbol{k}_{ \pm}$}

Some further properties of the vector fields $\boldsymbol{k}_{+}, \boldsymbol{J} \boldsymbol{k}_{+}$follow in the presence of integrability.

Proposition 3.8. Let $J=J_{g, \boldsymbol{k}_{ \pm}}$be an admissible complex structure. Then $\boldsymbol{k}_{+}$, $\boldsymbol{k}_{-}$are holomorphic vector fields with respect to $J$ if and only if they commute and are shear-free. 
Proof. We examine the Lie derivative formula $\left(\mathbf{L}_{\boldsymbol{k}_{ \pm}} J\right) a=\left[\boldsymbol{k}_{ \pm}, J a\right]-J\left[\boldsymbol{k}_{ \pm}, a\right]$, for $a$ taking values in the frame used in the proof of Theorem 1. For $a=\boldsymbol{k}_{+}$or $a=J \boldsymbol{k}_{+}$, its vanishing will occur if and only if $\boldsymbol{k}_{ \pm}$commute. For $a \in \Gamma(\mathcal{H})$, note first that $g\left(\left(\mathbf{L}_{\boldsymbol{k}_{ \pm}} J\right) a, \boldsymbol{k}_{ \pm}\right)=0$ by (11)i), so (8) implies that the projection of $\left(\mathbf{L}_{\boldsymbol{k}_{ \pm}} J\right) a$ to $\mathcal{V}$ vanishes. Next, one sees that $N\left(\boldsymbol{k}_{+}, \boldsymbol{x}_{+}\right)=\left(\mathbf{L}_{\boldsymbol{k}_{-}} J\right) \boldsymbol{x}_{+}+\left(\mathbf{L}_{\boldsymbol{k}_{+}} J\right) \boldsymbol{x}_{-}$. Thus repeating the calculation after (12) for each summand separately, we have, for example, $g\left(\left(\mathbf{L}_{\boldsymbol{k}_{+}} J\right) \boldsymbol{x}_{-}, \boldsymbol{x}_{ \pm}\right)=0$ if and only if the shear coefficients of $\boldsymbol{k}_{+}$vanish, or equivalently the projection of $\left(\mathbf{L}_{\boldsymbol{k}_{+}} J\right) \boldsymbol{x}_{-}$to $\mathcal{H}$ vanishes. Since $J\left(\mathbf{L}_{\boldsymbol{k}_{+}} J\right) \boldsymbol{x}_{-}=\left(\mathbf{L}_{\boldsymbol{k}_{+}} J\right) \boldsymbol{x}_{+}$, this is also equivalent to the statement that the restriction of $\mathbf{L}_{\boldsymbol{k}_{+}} J$ to $\mathcal{H}$ vanishes. Similar considerations lead to the vanishing of the restriction of $\mathbf{L}_{\boldsymbol{k}_{-}} J$ to $\mathcal{H}$.

Note that in our later examples, at times $\boldsymbol{k}_{+}$and $J \boldsymbol{k}_{+}$will not commute, and in one case they will not be shearfree.

\section{Kähler metrics induced by admissible metrics}

This section gives the construction of Kähler metrics on semi-Riemannian 4-manifolds we call admissible, which, in particular, possess an admissible complex structure.

\subsection{Admissible manifolds and metrics}

We first give the definition of manifold and metric admissibility. Recall in particular that a complex structure is admissible if (6), (7) and (11) hold. In this section and in others we will switch notations to $\boldsymbol{k}_{+}:=\boldsymbol{k}, \boldsymbol{k}_{-}:=\boldsymbol{t}$, so that $\mathcal{V}:=\operatorname{span}(\boldsymbol{k}, \boldsymbol{t})$.

Definition 4.1. An oriented semi-Riemannian manifold $(M, g)$ is called admissible if it is equipped with two vector fields $\boldsymbol{k}, \boldsymbol{t}$ for which

$$
\begin{aligned}
& \text { i] } J=J_{g, \boldsymbol{k}, \boldsymbol{t}} \text { is an admissible complex structure, } \\
& \text { ii] } \boldsymbol{t}=\ell \nabla \tau, \text { for } C^{\infty} \text { functions } \tau, \ell, \\
& \text { iii } \nabla(g(\boldsymbol{k}, \boldsymbol{t})), \nabla(g(\boldsymbol{k}, \boldsymbol{k})) \in \Gamma(\mathcal{V}) .
\end{aligned}
$$

The metric $g$ is also called admissible.

We will see in later sections many examples of admissible manifolds.

\subsection{The Kähler metrics}

We first give certain families of $J_{g, \boldsymbol{k}, t}$-invariant symplectic forms on admissible manifolds. Some of these forms, on Lorentzian manifolds with $\boldsymbol{k}$ null and geodesic, were first considered in [1] in analogy with wellknown contact forms on 3-manifolds.

Let $(M, g)$ be an admissible semi-Riemannian 4-manifold (Definition 4.1), with vector fields $\boldsymbol{k}, \boldsymbol{t}$. We consider 2 -forms on $M$ of the form

$$
\omega:=d\left(f(\tau) \boldsymbol{k}^{b}\right),
$$

where $\boldsymbol{k}^{b}=g(\boldsymbol{k}, \cdot)$, and $f$ is a smooth function defined on the range of $\tau$, for $\tau$ as in Definition 4.1ii]. Among the different choices of the "parameter function" $f$, our interest will lie mainly in the case where $f$ is affine in $\tau$, or else is $e^{\tau}$. As part of the following theorem, we will shortly show that the forms $\omega$ are $J_{g, \boldsymbol{k}, \boldsymbol{t}}$-invariant. It is thus natural to consider the tensor

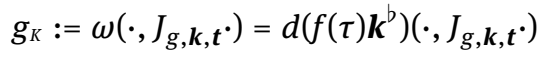

as a candidate for a Kähler metric. With $G$ as in (10), we have 
Theorem 3. Let $(M, g)$ be an admissible 4-manifold. Then $g_{\kappa}$, given in (19), is a Kähler metric on the region of $M$ where

$$
f_{\iota}<0 \text { and } f^{\prime} G / \ell-f d \boldsymbol{k}^{b}(\boldsymbol{k}, \boldsymbol{t})<0 .
$$

Proof. We first check that $g_{K}$ is $J_{g, \boldsymbol{k}, \boldsymbol{t}}$-invariant. It is enough to show the same for $\omega$, and we do so by evaluating it on $\boldsymbol{k}, \boldsymbol{t}, \boldsymbol{x}$, where $\boldsymbol{x} \in \Gamma(\mathcal{H})$. As there is clearly nothing to check for a pair of the form $a, J_{g, \boldsymbol{k}, \boldsymbol{t}} a$, we need not consider the case where both vectors lie in either of the $J$-invariant distributions $\mathcal{V}, \mathcal{H}$. For the case where one vector field is in $\mathcal{V}$ and the other in $\mathcal{H}$, we calculate $\omega(\boldsymbol{t}, \boldsymbol{x})=d\left(f \boldsymbol{k}^{b}\right)(\boldsymbol{t}, \boldsymbol{x})=f d \boldsymbol{k}^{b}(\boldsymbol{t}, \boldsymbol{x})=$ $f\left[g\left(\nabla_{\boldsymbol{t}} \boldsymbol{k}, \boldsymbol{x}\right)-g\left(\nabla_{\boldsymbol{x}} \boldsymbol{k}, \boldsymbol{t}\right)\right]=-f\left[g\left(\boldsymbol{k}, \nabla_{\boldsymbol{t}} \boldsymbol{x}\right)-g\left(\boldsymbol{k}, \nabla_{\boldsymbol{x}} \boldsymbol{t}\right)\right]=-f g(\boldsymbol{k},[\boldsymbol{t}, \boldsymbol{x}])=0$, where here $\nabla(g(\boldsymbol{k}, \boldsymbol{t})) \in \Gamma(\mathcal{V})$ and (11)i) were applied. An analogous calculation shows $\omega(\boldsymbol{k}, \boldsymbol{x})=g(\boldsymbol{k},[\boldsymbol{k}, \boldsymbol{x}])=0$ using $\nabla(g(\boldsymbol{k}, \boldsymbol{k})) \in \Gamma(\mathcal{V})$. As both calculations yield zero, $\omega$ and hence $g_{k}$ are $J_{g, k, t}$-invariant.

Now $\omega$ is clearly exact, and $J_{g, k, t}$ is integrable, so it remains to compute the region where $J_{g, k, t}$ is $\omega$-tame, yielding positive definiteness of $g_{k}$. It is clearly enough to check when

$$
g_{K}(a, a)=\omega(a, J a)>0
$$

for all vector fields $a$ of a $g_{K}$-orthogonal frame. We choose a frame $\boldsymbol{k}, \boldsymbol{t}, \boldsymbol{x}, \boldsymbol{y}=J_{g, \boldsymbol{k}, \boldsymbol{t}} \boldsymbol{x}$ with the latter two (possibly local) vector fields forming an oriented $g$-orthonormal frame for $\mathcal{H}$. We need only compute $\omega(\boldsymbol{x}, \boldsymbol{y})$ and $\omega(\boldsymbol{k}, \boldsymbol{t})$. The first is $\omega(\boldsymbol{x}, \boldsymbol{y})=f d \boldsymbol{k}^{b}(\boldsymbol{x}, \boldsymbol{y})=f\left(g\left(\nabla_{\boldsymbol{x}} \boldsymbol{k}, \boldsymbol{y}\right)-g\left(\nabla_{\boldsymbol{y}} \boldsymbol{k}, \boldsymbol{x}\right)\right)=-f$, by equation (4). For the second, we write

$$
\omega=f^{\prime} d \tau \wedge \boldsymbol{k}^{b}+f d \boldsymbol{k}^{b}
$$

Then $f^{\prime}\left(d \tau \wedge \boldsymbol{k}^{b}\right)(\boldsymbol{k}, \boldsymbol{t})=-f^{\prime}\left[g(\boldsymbol{k}, \boldsymbol{t})^{2}-g(\boldsymbol{t}, \boldsymbol{t}) g(\boldsymbol{k}, \boldsymbol{k})\right] / \ell=-f^{\prime} G / \ell$, so that $\omega(\boldsymbol{k}, \boldsymbol{t})=-f^{\prime} G / \ell+f d \boldsymbol{k}^{b}(\boldsymbol{k}, \boldsymbol{t})$. These expressions yield the inequalities in the theorem defining the region where $g_{K}$ is Kähler.

We will often describe a Kähler metric provided by the above theorem as induced by an admissible metric.

With $\alpha$ below given in (5), we have

Remark 4.2. The second inequality in (20), for the region where $g_{K}$ in (19) represents a Kähler metric, becomes

$$
\begin{aligned}
& f^{\prime} G / \ell<0 \text { if } \boldsymbol{k} \text { is geodesic of constant length, or } \\
& f^{\prime} G / \ell-f \alpha g(\boldsymbol{k}, \boldsymbol{t})<0 \text { if } \boldsymbol{k} \text { is null and strictly pre-geodesic, or } \\
& f^{\prime} G / \ell+f d_{\boldsymbol{t}}(g(\boldsymbol{k}, \boldsymbol{k}))<0 \text { if } \boldsymbol{k} \text { is Killing. }
\end{aligned}
$$

The first of these inequalities can be simplified to $f^{\prime} / \ell>0$ in the Lorentzian case, using (10).

In fact, $d \boldsymbol{k}^{b}(\boldsymbol{k}, \boldsymbol{t})=g\left(\nabla_{\boldsymbol{k}} \boldsymbol{k}, \boldsymbol{t}\right)-g\left(\nabla_{\boldsymbol{t}} \boldsymbol{k}, \boldsymbol{k}\right)=g\left(\nabla_{\boldsymbol{k}} \boldsymbol{k}, \boldsymbol{t}\right)-d_{\boldsymbol{t}}(g(\boldsymbol{k}, \boldsymbol{k})) / 2$ vanishes when $\boldsymbol{k}$ is geodesic with constant length, equals $\alpha g(\boldsymbol{k}, \boldsymbol{t})$ when $\boldsymbol{k}$ is a null and strictly pre-geodesic, and equals $-g\left(\boldsymbol{k}, \nabla_{\boldsymbol{t}} \boldsymbol{k}\right)-d_{\boldsymbol{t}}(g(\boldsymbol{k}, \boldsymbol{k})) / 2=$ $-d_{\boldsymbol{t}}(g(\boldsymbol{k}, \boldsymbol{k}))$ if $\boldsymbol{k}$ is Killing.

Remark 4.3. Let $J_{+}:=J$, and denote by $J_{-}$the almost complex structure of Remark 3.3, respecting the opposite orientation. It is easy to show that if $\boldsymbol{k}_{ \pm}$are shear-free, $g_{K}$ is ambihermitian, in the sense of [5]. In some cases, $g_{K}$ is also ambiKähler (e.g. when $g_{K}$ is an SKR metric as in Section 7).

\section{$4.3 g_{K}$ values on a frame}

We record a number of basic facts about $g_{K}$ inferred from Theorem 3.

Lemma 4.4. For a Kähler metric induced from an admissible semi-Riemannian metric,

$$
g_{K}(\mathcal{V}, \mathcal{H})=0, \quad g_{K}(\boldsymbol{k}, \boldsymbol{t})=0, \quad g_{K}(\boldsymbol{k}, \boldsymbol{k})=g_{K}(\boldsymbol{t}, \boldsymbol{t}),\left.\quad g_{K}\right|_{\mathcal{H}}=-\left.f \boldsymbol{l} g\right|_{\mathcal{H}} .
$$

Proof. The first equality was shown in the proof of Theorem 3. The second and third are obvious as $\omega$ is a 2-form. The fourth follows by comparing, with the help of (22), $\left.g_{K}\right|_{\mathcal{H}}(a, b)=f d \boldsymbol{k}^{b}(a, J b)=f\left(g\left(\nabla_{a} \boldsymbol{k}, J b\right)-\right.$ 
$\left.g\left(\nabla_{J b} \boldsymbol{k}, a\right)\right)$ and $-f\left(\left.g\right|_{\mathcal{H}^{-}}(a, b)\right.$ using (4), where $a, b$ are taken from an oriented orthonormal frame $\boldsymbol{x}, \boldsymbol{y}=J \boldsymbol{x}$ for $\mathcal{H}$.

Note that $g_{K}(\boldsymbol{k}, \boldsymbol{k})=g_{K}(\boldsymbol{t}, \boldsymbol{t})=f d \boldsymbol{k}^{b}(\boldsymbol{k}, \boldsymbol{t})-f^{\prime} G / \ell$ (see Theorem 3), which reduces to minus one of the expressions in the inequalities of Remark 4.2, in the case where $\boldsymbol{k}$ satisfies one of the corresponding assumptions given there. This formula will also be used later in the easily verifiable form

$$
\begin{aligned}
& g_{K}(\boldsymbol{k}, \boldsymbol{k})=g_{K}(\boldsymbol{t}, \boldsymbol{t})=-f\left(g([\boldsymbol{k}, \boldsymbol{t}], \boldsymbol{k})+d_{\boldsymbol{t}}(g(\boldsymbol{k}, \boldsymbol{k}))-f^{\prime} G / \ell,\right. \\
& \text { whenever } g(\boldsymbol{k}, \boldsymbol{t}) \text { is constant. }
\end{aligned}
$$

\subsection{Additional Kähler metrics and functions with a gradient in $\mathcal{V}$}

If $g$ is an admissible metric with distinguished shear-free vector fields $\boldsymbol{k}, \boldsymbol{t}$, then $g$ is also admissible relative to the pairs $a \boldsymbol{k}, \boldsymbol{t}$ and $\boldsymbol{k}, \boldsymbol{a t}$, whenever $a$ is a function with $\nabla a \in \Gamma(\mathcal{V})$. This can be shown easily by verifying i]iii] of Definition 4.1 for these pairs. Thus $g$ induces two new families of Kähler metrics for each such function $a$. The Kähler metrics relative to the pair $\boldsymbol{k}$, $a \boldsymbol{t}$ will have the same symplectic form as the original $g_{K}$ but a different complex structure. Note, however, that the function $a$ must, in fact, be functionally dependent on $\tau$ :

Proposition 4.5. On the domain of any associated Kähler metric in an admissible manifold, any function a with gradient in $\mathcal{V}$ must be locally a function of $\tau$ of Definition 4.1.

Proof. If $\nabla a=\alpha \boldsymbol{k}+\beta \boldsymbol{t}$ then $d a=\alpha \boldsymbol{k}^{b}+\beta \boldsymbol{t}^{b}$, so applying the exterior derivative on both sides gives $0=$ $d \alpha \wedge \boldsymbol{k}^{b}+\alpha d \boldsymbol{k}^{b}+d \beta \wedge \boldsymbol{t}^{b}+\beta d \boldsymbol{t}^{b}$. Applying this to the usual oriented orthonormal frame $\boldsymbol{x}, \boldsymbol{y}$ of $\mathcal{H}$ gives $0=\alpha d \boldsymbol{k}^{b}(\boldsymbol{x}, \boldsymbol{y})+\beta d \boldsymbol{t}^{b}(\boldsymbol{x}, \boldsymbol{y})$. Using $d \phi(a, b)=d_{a}(\phi(b))-d_{b}(\phi(a))-\phi([a, b])$ for $\phi=\boldsymbol{k}^{b}$ and $\phi=\boldsymbol{t}^{b}$ along the orthogonality of $\mathcal{V}$ and $\mathcal{H}$ yields $0=-\alpha \boldsymbol{k}^{b}([\boldsymbol{x}, \boldsymbol{y}])-\beta \boldsymbol{t}^{b}([\boldsymbol{x}, \boldsymbol{y}])=-\alpha \boldsymbol{l}^{\boldsymbol{k}}-\beta \boldsymbol{t}^{\boldsymbol{t}}$. But the twist function $\boldsymbol{t}^{\boldsymbol{t}}=0$ since $\iota^{\boldsymbol{t}}=g\left(\ell \nabla \tau, \nabla_{\boldsymbol{x}} \boldsymbol{y}-\nabla_{\boldsymbol{y}} \boldsymbol{x}\right)=-\ell\left(g\left(\nabla_{\boldsymbol{x}} \nabla \tau, \boldsymbol{y}\right)-g\left(\nabla_{\boldsymbol{y}} \nabla \tau, \boldsymbol{x}\right)\right)=0$ as the Hessian is symmetric. Thus, as $\iota^{\boldsymbol{k}}$ is nonzero on the domain of an associated Kähler metric, $\alpha=0$. So $d a=\beta t^{b}=\beta \ell d \tau$, hence $a$ is locally a function of $\tau$.

In particular, iii] of Definition 4.1 implies that for an admissible metric, on the domain of an induced Kähler metric, $g(\boldsymbol{k}, \boldsymbol{t})$ and $g(\boldsymbol{k}, \boldsymbol{k})$ are always functions of $\tau$.

\section{First-order properties of the induced Kähler metric}

In this section we will show that some properties of special admissible metrics are also shared by their induced Kähler metric, including admissibility itself. We will also examine other metric characteristics, such as completeness.

\subsection{Relations between the admissible and induced metrics}

We will occasionally employ the notation $\tau_{c}:=\tau-c$, for a constant $c$.

Proposition 5.1. Let $g$ be an admissible semi-Riemannian metric with $\boldsymbol{k}, \boldsymbol{t}$ geodesic vector fields of constant length, $g(\boldsymbol{k}, \boldsymbol{t})$ constant and $\ell=1$. If $g_{K}$ is the induced Kähler metric with $f(\tau)=\tau_{c}$, then the associated vector fields $\boldsymbol{k}, \boldsymbol{t}$ are geodesic and of constant length also with respect to $g_{K}$.

Proof. First, $\boldsymbol{k}$ has constant $g_{K}$-norm as $\boldsymbol{k}$ is geodesic of constant length, $f^{\prime}=\ell=1$ and $G$ is constant (see Remark 4.2 and the end of subsection 4.3). By Lemma 4.4, $\boldsymbol{t}$ also has constant $g_{K}$-norm. Let $\boldsymbol{x}, \boldsymbol{y}$ be a local 
orthonormal frame of $\left.g\right|_{H}$, and $\nabla^{K}$ denote the Levi-Civita connection of $g_{K}$. By the Koszul formula, the constant length of $\boldsymbol{k}$ implies $2 g_{K}\left(\nabla_{\boldsymbol{k}}^{K} \boldsymbol{k}, \boldsymbol{x}\right)=-2 g_{K}(\boldsymbol{k},[\boldsymbol{k}, \boldsymbol{x}])=0$, the last equality following as $[\boldsymbol{k}, \boldsymbol{x}]$ lies in $\mathcal{H}$, since admissible metrics satisfy the hypotheses of Theorem 1. The same holds for $\boldsymbol{x}$ replaced by $\boldsymbol{y}$, and clearly also $g_{K}\left(\nabla_{\boldsymbol{k}}^{K} \boldsymbol{k}, \boldsymbol{k}\right)=d_{\boldsymbol{k}}\left(g_{K}(\boldsymbol{k}, \boldsymbol{k})\right) / 2=0$.

The Koszul formula for $g$ also gives

$$
\begin{aligned}
& 0=-g\left(\nabla_{\boldsymbol{k}} \boldsymbol{k}, \boldsymbol{t}\right)=g(\boldsymbol{k},[\boldsymbol{k}, \boldsymbol{t}]), \\
& 0=-g\left(\nabla_{t} \boldsymbol{t}, \boldsymbol{k}\right)=g(\boldsymbol{t},[\boldsymbol{t}, \boldsymbol{k}])
\end{aligned}
$$

so $[\boldsymbol{k}, \boldsymbol{t}]$ is tangent to $\mathcal{H}$. Applying this, we see by invoking the Koszul formula once more, this time for $g_{K}$, that, as $\left.g_{K}\right|_{\mathcal{V}}$ is constant on $\boldsymbol{k}, \boldsymbol{t}, g_{K}\left(\nabla_{\boldsymbol{k}}^{K} \boldsymbol{k}, \boldsymbol{t}\right)=-g_{K}(\boldsymbol{k},[\boldsymbol{k}, \boldsymbol{t}])=0$ by the first relation in Lemma 4.4. Thus $g_{K}$ vanishes on any pair consisting of $\nabla_{\boldsymbol{k}}^{K} \boldsymbol{k}$ and any vector field in the $g_{K}$-orthogonal frame $\{\boldsymbol{k}, \boldsymbol{t}, \boldsymbol{x}, \boldsymbol{y}\}$, so $\nabla_{\boldsymbol{k}}^{K} \boldsymbol{k}=0$. A similar argument shows that $\nabla_{t}^{K} \boldsymbol{t}=0$.

A corresponding result when $\boldsymbol{k}$ is Killing is the following.

Proposition 5.2. Let $g$ be an admissible semi-Riemannian metric with $\boldsymbol{k}$ Killing, $\ell$, । functions of $\tau, g(\boldsymbol{k}, \boldsymbol{t})=0$ and $[\boldsymbol{k}, \boldsymbol{t}]=0$. Then for any induced Kähler metric $\boldsymbol{g}_{K}, \boldsymbol{k}$ is also $\boldsymbol{g}_{K}$-Killing.

We omit the proof, which consists of a systematic verification of the Killing field identity

$$
d_{\boldsymbol{k}}\left(g_{K}(a, b)\right)=g_{K}([\boldsymbol{k}, a], b)+g_{K}(a,[\boldsymbol{k}, b])
$$

when $a, b$ are taken from the usual frame vector fields $\boldsymbol{k}, \boldsymbol{t}, \boldsymbol{x}, \boldsymbol{y}=J \boldsymbol{x}$.

Examples where all these conditions are satisfied will be given in Section 7.

Finally, the shear condition (11)ii) of Theorem 1 holds also with the shears taken with respect to $g_{K}$. In fact, this follows from the following relations between shears with respect to an admissible metric $g$ and a Kähler metric $g_{K}$ it induces:

$$
\left(\nabla^{\nwarrow}\right)^{o} \boldsymbol{k}=\nabla^{o} \boldsymbol{k}, \quad\left(\nabla^{\nwarrow}\right)^{o} \boldsymbol{t}=\nabla^{o} \boldsymbol{t} .
$$

These relations can be verified by direct calculation. Of course, there is a more conceptual argument for the validity of the shear condition (11)ii) with respect to the Kähler metric. Namely, the proof of Theorem 1 shows that if conditions (11)i) hold, then the Nijenhuis tensor vanishes if and only if the shear condition (11)ii) is satisfied. This vanishing, along with (11)i), are of course metric independent conditions, so it follows that, assuming (11)i), if $J$ is integrable, condition (11)ii) must hold for any metric for which (6) and (7) hold. Now (7) certainly holds for the Riemannian metric $g_{K}$, while (6) also holds as it is independent of the metric.

\subsection{Repeated admissibility}

In the following proposition admissible metrics induce Kähler metrics that are also admissible.

Proposition 5.3. Let $g$ be an admissible semi-Riemannian metric on an oriented 4-manifold $M$ with distinguished vector fields $\boldsymbol{k}, \boldsymbol{t}$. Assume additionally that $g(\boldsymbol{k}, \boldsymbol{t})=0$ and $\ell, g(\boldsymbol{t}, \boldsymbol{t}), g([\boldsymbol{k}, \boldsymbol{t}], \boldsymbol{k})$ are all functions of $\tau$. For a given $f: \operatorname{Im} \tau \rightarrow \mathbb{R}$, let $g_{K}=g_{K}(g, \boldsymbol{k}, \boldsymbol{t}, f)$ be an induced Kähler metric on an appropriate region. Then $\left\{g_{K}, \boldsymbol{k}, \boldsymbol{t}\right\}$ is admissible and satisfies all the conditions mentioned above for $\mathrm{g}$. Thus there exists an infinite sequence of admissible Kähler metrics $g_{\kappa}^{(n)}, n \in \mathbb{N}$, with $g_{\kappa}^{(1)}:=g_{K}$, each defined by the quadruple $\left\{g_{\kappa}^{(n-1)}, \boldsymbol{k}, \boldsymbol{t}, f\right\}$ on an appropriate region $U^{(n)} \subset M$ (which may, however, be empty for some $n$ ).

Proof. To show that $g_{K}$ is admissible, taking into account that it is Riemannian, that $g_{K}(\boldsymbol{k}, \boldsymbol{t})=0$ and that (25) holds, it is enough to check $\nabla^{K}\left(g_{K}(\boldsymbol{k}, \boldsymbol{k})\right) \in \Gamma(\mathcal{V})$ and $\boldsymbol{t}=\ell_{K} \nabla^{K} \tau$ for some function $\ell_{K}$.

For the first of these, our assumptions together with (23) and the last sentence of Section 4 guarantee that $g_{K}(\boldsymbol{k}, \boldsymbol{k})$ is a function of $\tau$. Since $d_{\boldsymbol{x}} \tau=g(\boldsymbol{t} / \ell, \boldsymbol{x})=0$ for any $\boldsymbol{x} \in \Gamma(\mathcal{H})$, the claim follows. 
For the second claim, let $\boldsymbol{x}, \boldsymbol{y}$ be an orthonormal frame for $\left.g\right|_{\mathcal{H}}$. Since $g_{K}\left(\nabla^{K} \tau, b\right)=d \tau(b)=g(\nabla \tau, b)=$ $g(\boldsymbol{t}, b) / \ell$ and $g_{K}(\boldsymbol{t}, b)$ are both zero for $b=\boldsymbol{k}, \boldsymbol{x}, \boldsymbol{y}$, the only nonzero component of $\nabla^{K} \tau$ in the usual frame, which is $g_{K}$-orthogonal, is a multiple $\ell_{K}$ of $t$. Thus $g_{K}$ is admissible.

It remains to verify that the other assumptions of the proposition hold for $g_{K}$. We already know that $g_{K}(\boldsymbol{k}, \boldsymbol{t})=0$ and that $g_{K}(\boldsymbol{t}, \boldsymbol{t})=g_{K}(\boldsymbol{k}, \boldsymbol{k})$ is a function of $\tau$. For $\ell_{K}$ this is also clear since so are $g_{K}\left(\nabla^{K} \tau, \boldsymbol{t}\right)=$ $g(\boldsymbol{t}, \boldsymbol{t}) / \ell$ and $g_{K}(\boldsymbol{t}, \boldsymbol{t})$. Finally, expanding in the usual frame $[\boldsymbol{k}, \boldsymbol{t}]=a \boldsymbol{k}+\ldots$, clearly $g([\boldsymbol{k}, \boldsymbol{t}], \boldsymbol{k})=a g(\boldsymbol{k}, \boldsymbol{k})$, so $a$ is a function of $\tau(g(\boldsymbol{k}, \boldsymbol{k})$ is nowhere vanishing as $G \neq 0)$. Hence so is $g_{K}([\boldsymbol{k}, \boldsymbol{t}], \boldsymbol{k})=a g_{K}(\boldsymbol{k}, \boldsymbol{k})$.

One situation in which the domains of these successive Kähler metrics are never empty is described in the following proposition, whose proof is omitted.

Proposition 5.4. Under the conditions of Proposition 5.3, if additionally $p:=g(\boldsymbol{k}, \boldsymbol{k})>0$, fp is constant and fa is constant for a nonzero coefficient $a:=g([\boldsymbol{k}, \boldsymbol{t}], \boldsymbol{k}) / g(\boldsymbol{k}, \boldsymbol{k})$, then the domains of $g_{K}^{(n)}$ satisfy $U^{(n)} \subset U^{(n+1)}$ for all $n$.

\subsection{Completeness of geodesic vector fields}

Proposition 5.5. Let $\mathrm{g}$ be an admissible semi-Riemannian metric on a manifold $M$, with $\mathbf{k}$ a geodesic vector field of constant length. Suppose for some choice of a function $f(\tau)$, a complete Kähler metric $g_{K}$ is induced on all of $M$. Then any inextendible integral curve of $\mathbf{k}$ is defined on the whole real line if and only if $f^{\prime} G / \ell$ is bounded below on the curve.

Proof. According to [10, Proposition 3.4], a geodesic will be extendible beyond a finite interval of its parameter domain if and only if in some complete Riemannian metric the length of its tangents is bounded. Taking $g_{K}$ to be this metric, and the geodesic an integral curve of $\boldsymbol{k}$, extendibility will occur if and only if $g_{K}(\boldsymbol{k}, \boldsymbol{k})$ is bounded along it. But $g_{K}(\boldsymbol{k}, \boldsymbol{k})=-f^{\prime} G / \ell$ (see Remark 4.2).

Note that as $g_{K}(\boldsymbol{t}, \boldsymbol{t})=g_{K}(\boldsymbol{k}, \boldsymbol{k})$, if $\boldsymbol{t}$ is also geodesic, the same holds for its integral curves. If $g$ is Lorentzian or one of these vector fields is null, the same holds if $f^{\prime} / \ell$ is bounded above on the curve, as $G$ is negative (see (10)).

Examples of complete Kähler metrics induced from admissible manifolds appear in subsection 8.5.

\subsection{Admissible metrics inducing the same Kähler metric}

We briefly outline, without proof, a pair of circumstances in which two different semi-Riemannian metrics induce the same Kähler metric (for a given $f(\tau)$ ).

\subsubsection{Varying $\left.\mathbf{g}\right|_{\mathcal{H}}$}

On a given oriented 4-manifold fix two pointwise linearly independent vector fields $\boldsymbol{k}, \boldsymbol{t}$ spanning a distribution $\mathcal{V}$. Given a semi-Riemannian admissible metric $g$, consider a biconformal change of the form

$$
g=\left.\left.g\right|_{\mathcal{V}} \oplus g\right|_{\mathcal{H}} \rightarrow \tilde{g}=\left.\left.g\right|_{\mathcal{V}} \oplus \beta^{2} g\right|_{\mathcal{H}}
$$

for the usual $\mathcal{H}$ and a nowhere vanishing function $\beta$. One can show that $\tilde{g}$ is admissible and for fixed $f(\tau)$ one has $\tilde{g}_{K}=g_{K}$.

In Section 7 admissible metrics will be produced from a special type of Kähler metric that they, in turn, induce. In that setting there will be a canonical choice for $\left.g\right|_{\mathcal{H}}$ in such a biconformal class. 


\subsubsection{Varying $\left.\mathbf{g}\right|_{\mathcal{V}}$}

We now give a complementary change of metric, where $\left.g\right|_{\mathcal{H}}$ is fixed while $\left.g\right|_{\mathcal{V}}$ varies. Let $g$ be an admissible semi-Riemannian metric satisfying the hypotheses of Proposition 5.1. Let $\bar{g}=g+\varepsilon d \tau^{2}$ for small $\varepsilon>0$. The parameter $\varepsilon$ is only introduced so that $g$ and $\bar{g}$ have the same signature. Again one can show $\bar{g}$ is admissible, and for a given $f(\tau)$ the symplectic forms and admissible almost complex structures associated with $g$ and $\bar{g}$ coincide, so that $\bar{g}_{K}=g_{K}$.

\section{Ricci and scalar curvatures of $g_{K}$}

We give here formulas for the Ricci and scalar curvature of an induced Kähler metric $g_{\kappa}$ under certain assumptions, most importantly that the metrics conform to a certain bundle structure.

The Ricci form of a Kähler metric is given via

$$
\rho=-i \partial \bar{\partial} \log (\mu / v)=-\frac{1}{2} d J d \log (\mu / v),
$$

where $\mu$ is the volume form coefficient in a coordinate system and $v$ is the coefficient for the coordinate volume form in corresponding complex coordinates (cf. [33, \$1.4.3]). We apply this general formula to the case of the Kähler metric $g_{\kappa}$ associated to an admissible semi-Riemannian manifold, computed under certain assumptions detailed below. We give two formulas, one for the case that $\boldsymbol{k}$ is geodesic of constant length, the other for the case it is Killing. Examples fulfilling these assumptions will be given in Section 7 .

Proposition 6.1. Let $(M, g)$ be admissible, with admissible complex structure $J=J_{g, \boldsymbol{k}, \boldsymbol{t}}$. Assume that $M$ is an open set in the total space of a holomorphic line bundle over a two dimensional Kähler surface $(N, h)$ with holomorphic projection map $\pi$, and $\left.g\right|_{\mathcal{H}}=\pi^{\star} h$. Suppose $\boldsymbol{k}, \boldsymbol{t}$ commute and are shear-free, $\ell$ is a function of $\tau, \boldsymbol{k}$ is geodesic of constant nonzero length and $\boldsymbol{k}^{b}$ is locally a sum of an exact form and one vanishing on $v$.

Let $\omega^{h}=r d x \wedge d y$ be the Kähler form of $h$, expressed in local coordinates where $z=x+i y$ is a holomorphic coordinate on $N$. Then for any Kähler metric $g_{K}$ induced by $g$ and a parameter function $f(\tau)$, its Ricci form and scalar curvature are given, respectively, by

$$
\rho_{K}=-\frac{1}{2} d J d \log \left(-f f^{\prime} r \iota / \ell\right)
$$

and

$$
s_{K}=\frac{1}{2}\left(\left(\log \left(f^{\prime} f / \ell\right)\right)^{\prime} a f\right)^{\prime} /\left(f f^{\prime}\right)-\star\left[\frac{1}{2} \omega \wedge d J d \log (-r \iota)\right],
$$

where $\omega$, * are the Kähler form and Hodge star operator of $g_{\kappa}$, respectively, and a is the coefficient of $\boldsymbol{k}$ in the expression for $J d \tau$ as a linear combination of $\boldsymbol{k}$ and $d \tau$.

Note that we could write the second term in (27) much more explicitly by replacing $\omega$ via (22), but we have chosen not to, in order to keep the formula less cluttered. Also note that the assumption on $\boldsymbol{k}^{b}$ is fulfilled in the case it is a connection 1-form.

Proof. Given our assumptions on $\left.g\right|_{\mathcal{H}}$, the symplectic form $\omega$ takes the form

$$
\omega=f^{\prime} d \tau \wedge \boldsymbol{k}^{b}+f d \boldsymbol{k}^{b}=f^{\prime} d \tau \wedge \boldsymbol{k}^{b}-f i r d x \wedge d y,
$$

where we dropped pull-backs by $\pi$ from the notation. Thus the volume form is

$$
\mathrm{Vol}=\omega^{2} / 2=-f f^{\prime} r \iota d \tau \wedge \boldsymbol{k}^{b} \wedge d x \wedge d y,
$$

and $\mu=-f f^{\prime} r \iota$. 
Next, our assumptions, taken together with Proposition 3.8, mean that $\Xi:=\boldsymbol{k}-\boldsymbol{i} \boldsymbol{t}$ is a holomorphic vector field and $\psi:=\boldsymbol{k}^{b}+i \boldsymbol{t}^{b}$ a holomorphic 1 -form which evaluates to a nonzero constant on it. Employing $\psi$ together with $d z$ one computes the coordinate volume form coefficient to find, as $\boldsymbol{t}^{b}=\ell d \tau$ is exact because $\ell$ is a function of $\tau$, that up to a multiplicative constant, one can take $v=\ell$ (note that the assumption on $\boldsymbol{k}^{b}$ is used in this step).

Substituting in (26) gives the formula for the Ricci form.

To compute the scalar curvature, we first note that $J d \tau=J \boldsymbol{t}^{b} / \ell$ is a linear combination of $\boldsymbol{k}^{b}$ and $d \tau$, as it vanishes on $\mathcal{H}$. Substituting $\boldsymbol{k}$ and $\boldsymbol{t}$ in $J d \tau=a \boldsymbol{k}^{b}+b d \tau$ and solving the linear system for $a, b$, one easily sees, as the metric $g$ has constant values when evaluated on pairs from $\{\boldsymbol{k}, \boldsymbol{t}\}$, that $b$ is constant, while $a$, which also depends on $\ell$, is a function of $\tau$. Thus

$$
\begin{aligned}
\rho_{K} & =-\frac{1}{2} d J d \log \left(-f f^{\prime} r \iota / \ell\right)=-\frac{1}{2} d\left[\left(\log \left(f f^{\prime} / \ell\right)\right)^{\prime} J d \tau\right]-\frac{1}{2} d J d \log (-r \iota) \\
& =-\frac{1}{2} d\left[\left(\log \left(f f^{\prime} / \ell\right)\right)^{\prime}\left(a \boldsymbol{k}^{b}+b d \tau\right)\right]-\frac{1}{2} d J d \log (-r \iota) \\
& =-\frac{1}{2} d\left[\left(\log \left(f f^{\prime} / \ell\right)\right)^{\prime} a \boldsymbol{k}^{b}\right]-\frac{1}{2} d J d \log (-r \iota) \\
& =-\frac{1}{2}\left(\left(\log \left(f f^{\prime} / \ell\right)\right)^{\prime} a\right)^{\prime} d \tau \wedge \boldsymbol{k}^{b}-\frac{1}{2}\left(\log \left(f f^{\prime} / \ell\right)\right)^{\prime} a d \boldsymbol{k}^{b}-\frac{1}{2} d J d \log (-r \iota)
\end{aligned}
$$

Computing now $\omega \wedge \rho_{K}$, the contribution from the first two terms in the last line above combines to be

$$
\frac{1}{2}\left(\left(\log \left(f^{\prime} f / \ell\right)\right)^{\prime} a f\right)^{\prime} r \iota d \tau \wedge \boldsymbol{k}^{b} \wedge d x \wedge d y
$$

Since the scalar curvature is $S_{K}={ }^{\star}\left(\omega \wedge \rho_{K}\right)$, and ${ }^{\star} \operatorname{Vol}=1$, the result follows.

A special case of this formula for the scalar curvature occurs when $\ell=1$ and $f(\tau)=\tau_{c}$, as then $(a$ is constant and hence) the first term in (27) vanishes, giving

$$
s_{K}=-\star\left[\frac{1}{2} \omega \wedge d J d \log (-r l)\right]
$$

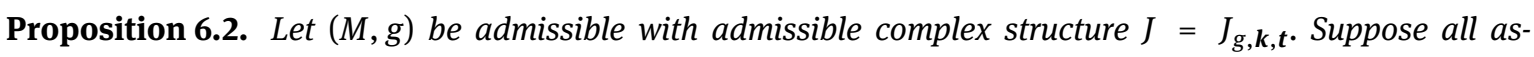
sumptions of Proposition 6.1 hold, except that $\boldsymbol{k}$ is not geodesic of constant length, but rather Killing. Assume $g(\boldsymbol{k}, \boldsymbol{t})=0, q:=g(\boldsymbol{t}, \boldsymbol{t}) \neq 0$ and $p:=g(\boldsymbol{k}, \boldsymbol{k}), \iota$ are, in addition to $\ell$, functions of $\tau$. Then for any Kähler metric $g_{K}$ induced by $g$ and a parameter function $f(\tau)$, its Ricci form and scalar curvature are given, respectively, by

$$
\rho_{K}=-\frac{1}{2} d J d \log \left(-f\left(f^{\prime}+f p^{\prime} / p\right) r \iota p / \ell\right)
$$

and

$$
s_{K}=\frac{\left(f a P^{\prime}\right)^{\prime}+2 f a P^{\prime} p^{\prime} / p}{f\left(f^{\prime}+f p^{\prime} / p\right)}-\frac{1}{2} \star[\omega \wedge d J d \log r],
$$

where $a=-q /(p \ell), P=-\log \left(-f\left(f^{\prime}+f p^{\prime} / p\right) p \iota / \ell\right) / 2$ and ${ }^{\star}$ is the Hodge star operator of $g_{K}$.

We omit the proof, which is somewhat more complex than the previous one but follows the same outline.

\section{Admissible Lorentzian metrics inducing SKR metrics}

In this section we begin the study of examples. We first recall the description of a special type of Kähler metric, called SKR, also known as a metric admitting a special Kähler-Ricci potential. These include many conformally-Einstein Kähler metrics. We then produce from such a metric, via an explicit ansatz, Lorentzian admissible metrics admitting a Killing field, which in some cases is also geodesic of constant length. These Lorentzian metrics in turn induce the initial SKR metric. 
A Killing potential $\tau$ on a Kähler manifold $\left(M, J, g_{K}\right)$ is, by definition, a smooth function $\tau$ such that $J \nabla^{K} \tau$ is a Killing vector field, where $\nabla^{K}$ denotes the Levi-Civita connection (or the gradient) with respect to the Kähler metric. We set

$$
v:=\nabla^{K} \tau, \quad u:=J v, \quad v:=\operatorname{span}(v, u), \quad \mathcal{H}:=\mathcal{V}^{\perp} .
$$

This potential $\tau$ is called a special Kähler-Ricci potential, and $g_{K}$ an SKR metric, if $\tau$ is nonconstant, and at each regular point of $\tau$, the nonzero tangent vectors in $\mathcal{H}$ are eigenvectors of both the Ricci endomorphism and the Hessian of $\tau$. We will often denote such metrics by $g_{\text {sR }}$. As mentioned above, they include many Kähler conformally Einstein metrics in dimension four (and all of those, in higher dimensions).

Theorem 18.1 in [13] gives the local classification of SKR metrics. It states that for any SKR metric, every regular point of $\tau$ has a neighborhood $U$ which is the domain of a biholomorphic isometry $\Psi$ to an open set in a holomorphic line bundle over a Kähler manifold $(N, h)$ with Kähler form $\omega^{h}$, equipped with the following metric, still denoted $g_{\text {SRR. }}$. There are in fact two metric forms, but we only give one, which we will call the irreducible form, as it describes metrics which are not Kähler local products. It is given as follows.

$$
g_{\text {SRR }} \text { is } \quad \frac{1}{Q} d \tau^{2}+\frac{Q}{a^{2}} \hat{u}^{2} \text { on } \mathcal{V}, \quad 2\left|\tau_{c}\right| \pi^{\star} h \text { on } \mathcal{H} .
$$

where $\tau_{c}:=\tau-c$ is as in Section 5.1 with $c$ a constant, $\tau$ is the push-forward of the Killing potential under the above biholomorphism, $a \neq 0$ is a constant, $Q$ is a function of $\tau$ which equals $g_{S R}(v, v)=g_{\mathscr{S R}}(u, u), \pi$ is the projection map from the line bundle to $N, \hat{u}$ is the one-form having value $a$ on $u$ and zero on $v$ and on lifts of vector fields on $N$, and $\mathcal{V}, \mathcal{H}$ are also obtained via pushing-forward the same-named distributions via the biholomorphism, with $\mathcal{V}$ being also the vertical distribution of the line bundle. In addition, $\mathcal{H}$ is the horizontal distribution for a Chern connection on the line bundle.

If $M$ is compact and not biholomorphic to $\mathbb{C P}^{m}$, it follows from [14] that a biholomorphic isometry $\Psi$ as above exists, with domain $M$, mapping onto a $\mathbb{C P}^{1}$-bundle over a Kähler manifold equipped with a canonical model metric. Furthermore, $\Psi$ maps the non-critical set of $\tau$ onto the total space of a line bundle minus its zero section. Finally, in the irreducible case the model metric still has the form (30) on this subset of the total space.

We record some known relations for SKR metrics, all immediate from or appearing in [13], some of which will be employed below. In these, $w, w^{\prime}$ denote horizontal lifts of vector fields on the base manifold $N$.

$$
\begin{aligned}
& \text { i) } g_{\mathbb{S R}}(u, v)=0, \quad \text { ii) } Q>0 \text { if } v \neq 0 \text { or } u \neq 0, \quad \text { iii }[u, v]=0, \\
& \text { iv) }[v, w]=0, \quad v)[u, w]=0, \quad v i)\left[w, w^{\prime}\right]^{\nu}=-2 \pi^{*} \omega^{h}\left(w, w^{\prime}\right) u .
\end{aligned}
$$

Given an SKR metric in dimension four of the form (30), our main objective is to obtain a procedure for finding an admissible Lorentzian metric $g$, defined on an appropriate subset $U$, with an induced Kähler metric satisfying $g_{K}=g_{\text {SR }}$ on $U$.

The construction is as follows. Set $\boldsymbol{k}:=u, \boldsymbol{t}:=-v$. Fix two of the three values of the metric on $\boldsymbol{k}, \boldsymbol{t}$ as follows: $g(\boldsymbol{k}, \boldsymbol{t}):=0$, while $g(\boldsymbol{t}, \boldsymbol{t}):=q$ is defined to be an arbitrarily chosen negative constant. Choose $p$ to be a function of a variable $\tau$ which is positive on $\{\tau>c\}$. Set $g(\boldsymbol{k}, \boldsymbol{k}):=p$, with $p$ now abusively denoting $p \circ \tau$. Define $\left.g\right|_{\mathcal{V}}$ by linear extension. Then define $\left.g\right|_{\mathcal{H}}:=\pi^{\star} h$. Finally, declare $g(\mathcal{V}, \mathcal{H})=0$.

In the following theorem we will take the domain of the biholomorphic isometry $\Psi$ to be the entire manifold under discussion, with the range contained in the total space of the line bundle. Our theorem is then stated as follows.

Theorem 4. Let $g_{S R}$ be an irreducible SKR metric on a complex manifold $(M, J)$ of real dimension four with special Kähler-Ricci potential $\tau$, such that the biholomorphic isometry $\Psi$ has domain $M$. Then there exists a Lorentzian metric on $U:=\{d \tau \neq 0\} \cap\{\tau>c\}$, which is isometric, via $\Psi$, to an admissible metric $g$ among those in the ansatz just described, whose distinguished vector fields are $\boldsymbol{k}$ and $\boldsymbol{t}$. 
Choosing $f(\tau):=\tau_{c} / p$, the metric $g$ along with $f$ induce a Kähler metric $g_{K}$ on the line bundle, whose isometric copy in $M$ (also denoted $g_{K}$ ) is defined on $U$ and satisfies

$$
g_{K}=g_{\text {SR }} \text { on } U \text {. }
$$

If $M$ is compact and not biholomorphic to $\mathbb{C P}^{2}$, then (after perhaps switching the sign of $\tau$ ) this Lorentzian metric is in fact defined on the set $U:=\{d \tau \neq 0\}$, which is open and dense in $M$.

Proof. We identify $U$ from now on with its image in the line bundle. It is easy to see that $g$ is Lorentzian and conditions (6) and (7) hold.

Conditions (11)i) follow immediately from (31)iv),v), the definition of $\boldsymbol{k}, \boldsymbol{t}$ and the fact that in the SKR setting, horizontal lifts of base vector fields span $\mathcal{H}$.

Condition ii) of (11) follows as the Koszul formula, applied to the expressions in (3), shows that $\boldsymbol{t}$ and $\boldsymbol{k}$ are shear-free.

Having checked i] of Definition 4.1 of admissibility, we now turn to ii]. First, note that setting $\ell:=-q / Q$ is well defined on $U$ as $Q$ is nonzero there. Now define $\tilde{\boldsymbol{t}}=\boldsymbol{t} / \ell$ (note that $\ell \neq 0$ ). We wish to show $\tilde{\boldsymbol{t}}=\nabla \tau$. The values of $g$ on the pairing of either of these vector fields with $\boldsymbol{x}, \boldsymbol{y}, \boldsymbol{k}$ is zero, since, for example $g(\tilde{\boldsymbol{t}}, \boldsymbol{x})=$ $g(\boldsymbol{t}, \boldsymbol{x}) / \ell=0=d_{\boldsymbol{x}} \tau=g(\boldsymbol{x}, \nabla \tau)$, and similarly $g(\tilde{\boldsymbol{t}}, \boldsymbol{k})=0$, while we have seen that $0=d_{\boldsymbol{k}} \tau=g(\boldsymbol{k}, \nabla \tau)$. Their values when paired with $\boldsymbol{t}$ are compared as follows: $g(\tilde{\boldsymbol{t}}, \boldsymbol{t})=q / \ell$ while $g(\nabla \tau, \boldsymbol{t})=d \tau(\boldsymbol{t})=g_{\text {SRR }}\left(\nabla^{\text {SR }} \tau, \boldsymbol{t}\right)=$ $g_{S R}(v, \boldsymbol{t})=g_{S R}(v,-v)=-Q=q / \ell$. This shows $\boldsymbol{t}=\ell \nabla \tau$.

To complete the proof that $g$ is admissible we need to verify iii] of Definition 4.1. Now $g(\boldsymbol{k}, \boldsymbol{t})$ is constant by the very definition of $g$. Also, $\nabla(g(\boldsymbol{k}, \boldsymbol{k})) \in \Gamma(\mathcal{V})$. This follows because $\nabla \boldsymbol{k}$ defines a map $\mathcal{H} \rightarrow \boldsymbol{k}^{\perp}$, as can be seen since for a vector field $\boldsymbol{x}$ with values in $\mathcal{H}, 2 g\left(\nabla_{\boldsymbol{x}} \boldsymbol{k}, \boldsymbol{k}\right)=d_{\boldsymbol{x}}(g(\boldsymbol{k}, \boldsymbol{k}))=d_{\boldsymbol{x}} p=p^{\prime} d_{\boldsymbol{x}} \tau=0$.

Next, we check that with $f$ given in the theorem, the induced $g_{K}$ is a Kähler metric on $U$. Note first that $q / \ell=-Q<0$ on $U$, while our $f$ is well defined and positive on $U$ and satisfies $f^{\prime} p+f p^{\prime}=(f p)^{\prime}=\tau_{c}^{\prime}=1$. Next, from (31)iii) we see that $[\boldsymbol{k}, \boldsymbol{t}]=0$, so that minus the expression (23), which is just the left hand side of (20) as $g(\boldsymbol{k}, \boldsymbol{t})=0$, evaluates to $f^{\prime} G / \ell+f d_{\boldsymbol{t}}(g(\boldsymbol{k}, \boldsymbol{k}))=(q / \ell)\left(f^{\prime} p+f p^{\prime}\right)=-Q$, which is negative on $U$. Also, applying (31)vi) and $\boldsymbol{k}=u$, we see that for the usual horizontal lifts $\boldsymbol{x}, \boldsymbol{y}=J \boldsymbol{x}$ we have $\iota=g(\boldsymbol{k},[\boldsymbol{x}, \boldsymbol{y}])=-2 \pi^{\star} \omega^{h}(\boldsymbol{x}, \boldsymbol{y}) p=$ $-2 \pi^{\star} h(y, y) p=-2 p<0$ on $U$. Hence $f l<0$ on $U$. Thus, by Theorem 3, $g$ induces a metric $g_{K}$ on $U$ which is Kähler with respect to $J$, as clearly $J=J_{g, \boldsymbol{k}, \boldsymbol{t}}$.

We now show that $g_{K}=g_{\Im R}$ on $U$. In fact, $g_{K}(\boldsymbol{t}, \boldsymbol{t})=g_{K}(\boldsymbol{k}, \boldsymbol{k})=-\left(f^{\prime} G / \ell+f d_{t} p\right)=Q$ as we have just seen, i.e. this value is equal to $g_{\text {XR }}(-v,-v)=g_{\text {SR }}(u, u)$, while $g_{K}(\boldsymbol{k}, \boldsymbol{t})=0=g_{\text {צRR }}(u,-v)$. Thus $\left.g_{K}\right|_{\mathcal{V}}=\left.g_{\text {SRR }}\right|_{\mathcal{V}}$. On the other hand, $\left.g_{K}\right|_{\mathcal{H}}=-\left.f ı\right|_{\mathcal{H}}=-f(-2 p) \pi^{\star} h=2 \tau_{c} \pi^{\star} h=2\left|\tau_{c}\right| \pi^{\star} h=\left.g_{\mathscr{S R}}\right|_{\mathcal{H}}$ on $U$. As $g_{K}(\mathcal{V}, \mathcal{H})=0=g_{\mathscr{S R}}(\mathcal{V}, \mathcal{H})$, the first equality following from Lemma 4.4, the two Kähler metrics indeed coincide.

Finally, if $M$ is compact and not biholomorphic to $\mathbb{C P}^{2}$, we know that the non-critical set of $\tau$ is mapped via $\Psi$ onto the line bundle minus its zero section, and we wish to show the former set is $U$. It was shown in [14] that for an SKR metric on a compact manifold, the range of $\tau$ is a closed interval and $c$ is not in its interior. As choosing the sign of $\tau$ determines that of $c$, one can thus always arrange, when the manifold is compact, that $\tau>c$ everywhere. Then, from $U$ 's definition it follows that $U$ is exactly the non-critical set of $\tau$. As $\tau$ is a Killing potential, it is known that the latter set is open and dense in $M$ (cf. [14]).

This completes the proof.

Note that it is possible to show that $\boldsymbol{k}$ is $g$-Killing and, if $p$ is a positive constant, also g-geodesic, while $\boldsymbol{t}$ is always $g$-geodesic.

It is worth mentioning that one can modify the ansatz slightly so that the Lorentzian metric will admit a timelike Killing field. 


\section{Kähler metrics induced by Lorentzian warped products}

\subsection{The construction}

In this section we construct Kähler 4-manifolds from admissible Lorentzian 4-manifolds, in the sense of Definition 4.1, which are warped products.

Theorem 5. Let $(N, \bar{g})$ be a Riemannian 3-manifold with a unit length vector field $\overline{\boldsymbol{k}}$, whose flow is geodesic, shear-free, and has a nowhere vanishing twist function. Let $w(t)$ be a smooth positive function on $\mathbb{R}$ satisfying $w^{\prime} / w>-1$. Then $(\mathbb{R} \times N, g, \boldsymbol{k}, \nabla t)$ is admissible with respect to a chosen orientation, where $g$ is the Lorentzian warped product

$$
g:=-d t^{2}+w^{2} \bar{g}
$$

and $\boldsymbol{k}:=\partial_{t}+\overline{\boldsymbol{k}} / w$. The metric $g$ then induces a Kähler metric on $\mathbb{R} \times N$.

Note that in the expression for $\boldsymbol{k}$, the notation $\overline{\boldsymbol{k}}$ refers to the obvious lift of this vector field from $N$ to $\mathbb{R} \times N$.

Proof. We verify the admissible properties of Definition 4.1. To begin with, our vector field $\boldsymbol{k}$ is clearly $g$-null, and pre-geodesic:

$$
\nabla_{\boldsymbol{k}} \boldsymbol{k}=\frac{w^{\prime}}{w} \boldsymbol{k}
$$

(For the properties satisfied by the Levi-Civita connection of warped products, see [27, Proposition 35, p. 206].) Next, set $\boldsymbol{t}:=\nabla t=-\partial_{t}$ and observe that

$$
g(\boldsymbol{t}, \boldsymbol{t})=-1, \quad \nabla_{\boldsymbol{t}} \boldsymbol{t}=0, \quad g(\boldsymbol{k}, \boldsymbol{t})=\boldsymbol{k}(t)=1 .
$$

We now establish relations between the shear, and later also twist functions of $\boldsymbol{k}$ and $\overline{\boldsymbol{k}}$. In what follows, the shear coefficients and twist function (up to sign) of $\overline{\boldsymbol{k}}$ will be denoted by $\bar{\sigma}_{1}, \bar{\sigma}_{2}$ and $\bar{\imath}$, respectively, while those of $\boldsymbol{k}$ will be denoted by $\sigma_{1}, \sigma_{2}$ and $\iota$.

Let $\{\overline{\boldsymbol{x}}, \overline{\boldsymbol{y}}\}$ be an ordered local $\bar{g}$-orthonormal frame of $\overline{\boldsymbol{k}}^{\perp \bar{s}} \subset T N$, whose ordering is chosen so that $\overline{\boldsymbol{l}}$ is negative. Lifting these vector fields trivially to $\mathbb{R} \times N$, the vector fields $\boldsymbol{x}:=\overline{\boldsymbol{x}} / w$ and $\boldsymbol{y}:=\overline{\boldsymbol{y}} / w$ form a $g$ orthonormal frame for the (spacelike) distribution $\mathcal{H}=\operatorname{span}(\boldsymbol{k}, \boldsymbol{t})^{\perp_{g}}$. Furthermore, $\nabla_{\boldsymbol{x}} \boldsymbol{k}=\frac{1}{w^{2}}\left(w^{\prime} \overline{\boldsymbol{x}}+\bar{\nabla}_{\overline{\boldsymbol{x}}} \overline{\boldsymbol{k}}\right)$, $\nabla_{\boldsymbol{y}} \boldsymbol{k}=\frac{1}{w^{2}}\left(w^{\prime} \overline{\boldsymbol{y}}+\overline{\nabla_{\bar{y}}} \overline{\boldsymbol{k}}\right)$, where $\bar{\nabla}$ is the Levi-Civita connection of $\bar{g}$. That $\boldsymbol{k}$ is shear-free now follows from this and the fact that $\overline{\boldsymbol{k}}$ is shear-free in $(N, \bar{g})$ :

$$
2 \sigma_{1}=g\left(\nabla_{\boldsymbol{y}} \boldsymbol{k}, \boldsymbol{y}\right)-g\left(\nabla_{\boldsymbol{y}} \boldsymbol{k}, \boldsymbol{x}\right)=\frac{2}{w} \bar{\sigma}_{1}=0,
$$

and a similar relation for $\sigma_{2}$. It is likewise verified that $\boldsymbol{t}$ is also shear-free, since $\nabla_{\boldsymbol{x}} \boldsymbol{t}=-\left(w^{\prime} / w\right) \boldsymbol{x}$ and $\nabla_{\boldsymbol{y}} \boldsymbol{t}=$ $-\left(w^{\prime} / w\right) \boldsymbol{y}$. Thus, the almost complex structure $J:=J_{g, \boldsymbol{k}, \boldsymbol{t}}$ compatible with the orientation for which $J \boldsymbol{x}:=$ $\boldsymbol{y}, J \boldsymbol{y}:=-\boldsymbol{x}$, satisfies the shear condition $J \nabla^{o} \boldsymbol{k}=\nabla^{o} \boldsymbol{t}=0$.

Furthermore, as $\nabla_{\boldsymbol{k}} \boldsymbol{t}=-\frac{w^{\prime}}{w^{2}} \overline{\boldsymbol{k}}$ and $\nabla_{\boldsymbol{t}} \boldsymbol{k}=0$, condition (17) also holds. Thus all conditions of subsection 3.3 hold, so that (14) and therefore (11)i) hold. Thus by Theorem 1, $J$ is integrable. Also all conditions in Definition 4.1 hold, so $(\mathbb{R} \times N, g, \boldsymbol{k}, \boldsymbol{t})$ is therefore admissible.

To complete the proof, we apply Theorem 3 to show that $g$ induces a Kähler metric on $\mathbb{R} \times N$. Recalling the definition of $\iota$ in (4), observe that (up to sign), $\iota$ of $\boldsymbol{k}$ in $(\mathbb{R} \times N, g)$ is related to that of $\overline{\boldsymbol{k}}$ in $(N, \bar{g})$ by

$$
\iota=g\left(\nabla_{\boldsymbol{y}} \boldsymbol{k}, \boldsymbol{x}\right)-g\left(\nabla_{\boldsymbol{x}} \boldsymbol{k}, \boldsymbol{y}\right)=\frac{1}{w} \bar{\imath}<0 .
$$

Now choose $f(t)=e^{t}$. Since $w^{\prime} / w>-1$ and $G=-\ell=-1$,

$$
f \iota=e^{t} \iota<0, \quad f^{\prime} G / \ell-f\left(w^{\prime} / w\right) g(\boldsymbol{k}, \boldsymbol{t})=-e^{t}\left(1+\left(w^{\prime} / w\right)\right)<0,
$$

so that $g_{K}=d\left(e^{t} \boldsymbol{k}^{b}\right)(\cdot, J \cdot)$ will be a Kähler metric on all of $\mathbb{R} \times N$. 
In [2] it is shown that (for other functions $f(t)$ ) one can produce Kähler-Einstein metrics from this construction. By Lemma 2.5, this result immediately yields:

Corollary 8.1. Let $(N, \bar{g})$ be a Riemannian 3-manifold and $X$ a unit length vector field whose flow is complete, geodesic, and shear-free. If $\operatorname{Ric}_{\bar{g}}(X, X)>0$, then $\mathbb{R} \times N$ admits a Kähler metric as in Theorem 5 .

We now present some concrete realizations of Theorem 5.

\subsection{A Kähler metric via the direct product on $\mathbb{R} \times \mathbb{S}^{3}$}

Let $\bar{g}$ denote the standard round metric on the 3-sphere $\mathbb{S}^{3}$. On $\mathbb{S}^{3}$, let $\overline{\boldsymbol{k}}$ denote the unit length Killing vector field tangent to the Hopf fibration. By Lemma 2.6, the flow of $\overline{\boldsymbol{k}}$ is geodesic and shear-free in $\left(\mathbb{S}^{3}, \bar{g}\right)$, and its twist function $\iota$ satisfies

$$
\iota^{2}=2 \operatorname{Ric}_{\bar{g}}(\overline{\boldsymbol{k}}, \overline{\boldsymbol{k}})=2 .
$$

Applying Theorem 5 with $w=1, \boldsymbol{k}=\partial_{t}+\overline{\boldsymbol{k}}$, and $\boldsymbol{t}=\nabla t$, we conclude that $\left(\mathbb{R} \times \mathbb{S}^{3},-d t^{2} \oplus \overline{\boldsymbol{g}}, \boldsymbol{k}, \boldsymbol{t}\right)$ is admissible and induces a Kähler metric on $\mathbb{R} \times \mathbb{S}^{3}$. It will turn out that this Kähler metric, defined with $f(t)=e^{t}$, is flat (see [2]).

\subsection{A Kähler metric on de Sitter spacetime}

Four-dimensional de Sitter spacetime is the warped product $\left(\mathbb{R} \times \mathbb{S}_{r}^{3},-d t^{2}+w^{2} \bar{g}\right)$, where $w(t)=r^{2} \cosh ^{2}(t / r)$ and $r>0$ is the radius of $\mathbb{S}_{r}^{3}$. This metric is both globally hyperbolic and geodesically complete (see [7, p. 183-4]). For $r \geq 2, w^{\prime} / w>-1$ on the entire manifold (if $r<2$, then on an open subset). Thus, with $\boldsymbol{k}=\partial_{t}+\overline{\boldsymbol{k}} / w$ and $\boldsymbol{t}=\nabla t$, Theorem 5 applies.

\subsection{Kähler metrics on $\mathbb{R} \times \mathbb{R}^{3}$}

Using Theorem 5 once again, a family of Kähler manifolds will now be constructed on $\mathbb{R}^{4}$ out of the following distinguished class of Lorentzian 4-manifolds:

Definition 8.2. A four-dimensional standard pp-wave is the Lorentzian manifold $\left(\mathbb{R}^{4}, g\right)$ with coordinates $(u, v, x, y)$ and with $g$ given by

$$
g=H(u, x, y) d u \otimes d u+d u \otimes d v+d v \otimes d u+d x \otimes d x+d y \otimes d y,
$$

for $H$ smooth. If $H$ is quadratic in $x$ and $y$, then $\left(\mathbb{R}^{4}, g\right)$ is called a plane wave.

Standard pp-waves originated in gravitational physics and have been intensely studied therein; see, e.g., [23] and [30], as well as [17] and [7, Chapter 13]. They are distinguished by the fact that $\partial_{v}=\nabla u$ is a parallel null vector field. Now let $k, h: \mathbb{R}^{4} \longrightarrow \mathbb{R}$ be two smooth functions independent of $v$ and consider the following null vector field $\boldsymbol{z}$ on $\left(\mathbb{R}^{4}, g\right)$ :

$$
\boldsymbol{z}:=\frac{1}{2}\left(H+k^{2}+h^{2}\right) \partial_{v}-\partial_{u}+k \partial_{x}+h \partial_{y} .
$$

Observe that $\partial_{v}$ and $z$ are pointwise linearly independent, that $g\left(\partial_{v}, z\right)=-1$, and finally that the pair of vector fields

$$
\boldsymbol{x}:=k \partial_{v}+\partial_{x}, \quad \boldsymbol{y}:=h \partial_{v}+\partial_{y}
$$

are orthonormal and span the distribution $\mathcal{H}=\operatorname{span}\left(\partial_{v}, \boldsymbol{z}\right)^{\perp}$, which is spacelike. Note also that the twist function of $\boldsymbol{z}$ does not, in general, vanish, as $\boldsymbol{l}^{z}$ is

$$
g(\boldsymbol{z},[\boldsymbol{x}, \boldsymbol{y}])=g\left(\boldsymbol{z},\left(-k_{y}+h_{x}\right) \partial_{v}\right)=k_{y}-h_{x} .
$$


Because $\partial_{v}=\nabla u$, the hypersurfaces $S_{u}:=\{u=$ const. $\}$ are integral submanifolds of the orthogonal complement $\partial_{v}^{\perp} \subset T \mathbb{R}^{4}$; because $\partial_{v}$ is null, it is tangent to these submanifolds. Now fix any $u_{0}$ and consider the hypersurface $S_{u_{0}} \cong \mathbb{R}^{3}$ with global coordinates $\{v, x, y\}$. Set

$$
\overline{\boldsymbol{k}}:=\left.\partial_{v}\right|_{S_{u_{0}}}, \quad \overline{\boldsymbol{x}}:=\left.\boldsymbol{x}\right|_{S_{u_{0}}}, \quad \overline{\boldsymbol{y}}:=\left.\boldsymbol{y}\right|_{S_{u_{0}}}
$$

and define a Riemannian metric $\bar{g}$ on $S_{u_{0}}$, by giving its orthonormal coframe $\left\{\overline{\boldsymbol{k}}^{\bar{b}}, \overline{\boldsymbol{x}}^{\bar{b}}, \overline{\boldsymbol{y}}^{\bar{b}}\right\}$, which is $\bar{g}$-dual to $\{\overline{\boldsymbol{k}}, \overline{\boldsymbol{x}}, \overline{\boldsymbol{y}}\}$. It is given by

$$
\bar{g}(\overline{\boldsymbol{k}}, \cdot):=-\left.g(\boldsymbol{z}, \cdot)\right|_{S_{u_{0}}}, \quad \bar{g}(\overline{\boldsymbol{x}}, \cdot):=\left.\boldsymbol{g}(\boldsymbol{x}, \cdot)\right|_{S_{u_{0}}}, \quad \bar{g}(\overline{\boldsymbol{y}}, \cdot):=\left.g(\boldsymbol{y}, \cdot)\right|_{S_{u_{0}}},
$$

so that $\{\overline{\boldsymbol{k}}, \overline{\boldsymbol{x}}, \overline{\boldsymbol{y}}\}$ is a global orthonormal frame for $\left(\mathbb{R}^{3}, \bar{g}\right)$. (This Riemannian metric is derived from a wellknown construction; see, e.g., [23]. Note that it does not depend on $H$.) We now have:

Proposition 8.3. On $\left(\mathbb{R}^{3}, \bar{g}\right)$ with $\bar{g}$ given by (39), the vector field $\overline{\boldsymbol{k}}$ given in (38) is a unit length Killing vector field. If $h_{x}-k_{y}$ is nowhere vanishing and $w$ is a smooth positive function satisfying $w^{\prime} / w>-1$, then $\left(\mathbb{R} \times \mathbb{R}^{3},-d t^{2}+\right.$ $\left.w^{2} \bar{g}, \overline{\boldsymbol{k}} / w+\partial_{t}, \nabla t\right)$ is admissible and induces a Kähler metric on $\mathbb{R}^{4}$.

Proof. We first show that $\overline{\boldsymbol{k}}$ is a unit length Killing vector field, via Lemma 2.6. That $\overline{\boldsymbol{k}}$ has unit length with respect to $\bar{g}$ is clear, since $\bar{g}(\overline{\boldsymbol{k}}, \overline{\boldsymbol{k}})=-g(\boldsymbol{z}, \overline{\boldsymbol{k}})=1$. Letting $\bar{\nabla}$ denote the Levi-Civita connection of $\left(\mathbb{R}^{3}, \bar{g}\right)$, it follows in particular that $\bar{g}\left(\bar{\nabla}_{\overline{\boldsymbol{k}}} \overline{\boldsymbol{k}}, \overline{\boldsymbol{k}}\right)=0$. Next, observe that

$$
\bar{g}\left(\overline{\nabla_{\overline{\boldsymbol{k}}}} \overline{\boldsymbol{k}}, \overline{\boldsymbol{x}}\right)=-\bar{g}(\overline{\boldsymbol{k}},[\overline{\boldsymbol{k}}, \overline{\boldsymbol{x}}])=\left.g\left(\boldsymbol{z},\left[\partial_{v}, \boldsymbol{x}\right]\right)\right|_{S_{u_{0}}}=0,
$$

where we have used $[\overline{\boldsymbol{k}}, \overline{\boldsymbol{x}}]=\left.\left[\partial_{v}, \boldsymbol{x}\right]\right|_{S_{u_{0}}}$ because $\left[\partial_{v}, \boldsymbol{x}\right]$ is tangent to $S$, and also $\left[\partial_{v}, \boldsymbol{x}\right]=0$. Likewise, $\bar{g}\left(\bar{\nabla}_{\overline{\boldsymbol{k}}} \overline{\boldsymbol{k}}, \overline{\boldsymbol{y}}\right)=0$, so that $\bar{\nabla}_{\overline{\boldsymbol{k}}} \overline{\boldsymbol{k}}=0$, hence $\overline{\boldsymbol{k}}$ has geodesic flow in $\left(\mathbb{R}^{3}, \bar{g}\right)$. That $\overline{\boldsymbol{k}}$ is both divergence-free and shear-free is similarly determined. Being unit length, geodesic, divergence-free, and shear-free, it follows by Lemma 2.6 that $\overline{\boldsymbol{k}}$ is a unit length Killing vector field on the Riemannian 3-manifold $\left(\mathbb{R}^{3}, \bar{g}\right)$. Finally, we show that the twist function of $\overline{\boldsymbol{k}}$ is nowhere vanishing, provided that $k_{y} \neq h_{x}$ at any point. This follows from our particular choice of $z$, namely, that its twist function is nowhere vanishing:

$$
\bar{\imath}:=\bar{g}(\overline{\boldsymbol{k}},[\overline{\boldsymbol{x}}, \overline{\boldsymbol{y}}])=-\left.g(\boldsymbol{z},[\boldsymbol{x}, \boldsymbol{y}])\right|_{S_{u_{0}}}=\left.\left(h_{x}-k_{y}\right)\right|_{S_{u_{0}}}
$$

Applying now the contents of Theorem 5, the proof is complete.

Some of the Kähler metrics induced by such "truncated" pp-wave metrics as in this proposition, as well as some of those induced directly from plane waves (see next section), are shown in [2] to be central metrics [24]. More precisely, the determinant of their Ricci endomorphism vanishes. Other such metrics are shown there to be Kähler-Einstein.

\subsection{Complete induced Kähler metrics}

We show here that one can in some instances obtain complete Kähler metrics via the construction of Theorem 5.

To proceed we choose a compact 3-manifold $N$ with the properties in Theorem 5 and consider on $M=N \times \mathbb{R}$ a Kähler metric $g_{K}$ induced according to that theorem but defined via an arbitrary function $f$. Its domain is then computed (via Theorem 3, the sign information for $\bar{l}$ and $w$ along with (33), Remark 4.2 and (32)) to be

$$
f>0, \quad(f w)^{\prime} / w>0 .
$$

Let $\hat{\boldsymbol{k}}$ denote the 1 -form that is 1 on $\boldsymbol{k}$ and zero on the other frame fields, and have $\hat{\boldsymbol{t}}, \hat{\boldsymbol{x}}, \hat{\boldsymbol{y}}$, where $\boldsymbol{x}, \boldsymbol{y}$ form an oriented orthonormal frame for $\mathcal{H}$, denote analogous 1 -forms. Similarly denote by $\hat{\overline{\boldsymbol{k}}}$ the pull-back from $N$ 
of the one-form dual in the same sense to $\overline{\boldsymbol{k}}$. Now $d t=\hat{\boldsymbol{k}}-\hat{\boldsymbol{t}}$, while $(\hat{\boldsymbol{k}}+\hat{\boldsymbol{t}})(\overline{\boldsymbol{k}} / w)=2$ so that the Kähler metric takes the form

$$
\begin{aligned}
g_{K} & =c\left(\hat{\boldsymbol{k}}^{2}+\hat{\boldsymbol{t}}^{2}\right)-f_{l}\left(\hat{\boldsymbol{x}}^{2}+\hat{\boldsymbol{y}}^{2}\right) \\
& =(c / 2)\left((\hat{\boldsymbol{k}}-\hat{\boldsymbol{t}})^{2}+(\hat{\boldsymbol{k}}+\hat{\boldsymbol{t}})^{2}\right)-f l\left(\hat{\boldsymbol{x}}^{2}+\hat{\boldsymbol{y}}^{2}\right) \\
& =(c / 2)\left(d t^{2}+(2 w \hat{\overline{\boldsymbol{k}}})^{2}\right)-f w^{-1} \bar{l}\left(\hat{\boldsymbol{x}}^{2}+\hat{\boldsymbol{y}}^{2}\right)
\end{aligned}
$$

for $c=g_{K}(\boldsymbol{k}, \boldsymbol{k})=(f w)^{\prime} / w$. It follows that $g_{K}$ has the form $d s^{2}+g_{s}$ where $s=\int \sqrt{c / 2} d t$ and $g_{s}$ form a family of metrics on $N$. As $N$ is compact, such metrics are complete on $I \times N$ whenever $I=(\inf s, \sup s)=\mathbb{R}$ (cf. [3, Lemma 33]). The latter can easily be arranged with an appropriate choice of $f$ and $w$.

\section{A Kähler metric induced by a gravitational plane wave}

In Proposition 8.3 we used three-dimensional submanifolds of pp-waves (Definition 8.2) to construct Kähler metrics on admissible four-dimensional Lorentzian warped products. Key to this construction was the null vector field (35) of a pp-wave, which was used to define the Riemannian metric (39). In this example we will construct a Kähler metric which will be induced directly from an admissible 4-dimensional pp-wave (in fact, a plane wave), without first constructing a Riemannian metric on a hypersurface.

Proposition 9.1. Let $\left(\mathbb{R}^{4}, g\right)$ be a pp-wave with $H(u, x, y)=-x^{2}-y^{2}$ and let $\boldsymbol{z}$ be the null vector field (35) with $k(u, x, y)=-y$ and $h(u, x, y)=x$. Then $\left(\mathbb{R}^{4}, g, z, \nabla u\right)$ is admissible and induces a Kähler metric on $\mathbb{R}^{4}$.

Proof. $\boldsymbol{z}=-\partial_{u}-y \partial_{x}+x \partial_{y}$ and $\nabla u=\partial_{v}$ are everywhere linearly independent and the orthonormality of $\boldsymbol{x}, \boldsymbol{y}$ of (36) shows that $\mathcal{H}:=\operatorname{span}(\nabla u, z)^{\perp}$ is spacelike, as required in (7). For $\boldsymbol{k}_{+}:=\boldsymbol{z}$ and the parallel field $\boldsymbol{k}_{-}:=\nabla u$, (15)a) holds, as well as (16) and condition (17), the latter since $\nabla_{\partial_{v}} \boldsymbol{z}=\nabla_{\boldsymbol{z}} \partial_{v}=0$. Thus by Remark 3.5, (11)i) holds, as can also be seen directly from the bracket relations of these vector fields with $\boldsymbol{x}, \boldsymbol{y}$, which also imply using (3) that $z, \nabla u$ are shear-free. Thus the associated almost complex structure is integrable. Next, $g(\boldsymbol{z}, \boldsymbol{z})=0$ and $g(\nabla u, \boldsymbol{z})=-1$, so $\left(\mathbb{R}^{4}, g, \boldsymbol{z}, \nabla u\right)$ is admissible. Now set $f=e^{u}$ and note that $\boldsymbol{l}^{\boldsymbol{z}}=k_{y}-h_{x}=-2$. That $g_{K}=d\left(e^{u} \boldsymbol{z}^{b}\right)(\cdot, J \cdot)$ is Kähler on $\mathbb{R}^{4}$ now follows from Theorem 3 and Remark 4.2, as $f l=-2 e^{u}<0$ and $f^{\prime} G / \ell=-e^{u}<0$.

It can be shown that $z$ is $g_{K}$-conformal (in fact $g_{K}$-homothetic) while $\nabla u$ is $g_{K}$-Killing. It is shown in [2] that $g_{K}$ has vanishing Ricci determinant.

\section{Kähler metrics on Petrov type $D$ spacetimes}

In this section we present two examples of Lorentzian 4-manifolds that fall outside the admissible category, and a third admissible one. These examples belong to the class of Lorentzian 4-manifolds of Petrov Type D, and include the Kerr spacetime. The relationship between such spacetimes and Kähler geometry was considered by Flaherty [16], who was lead to construct mostly modified-Kähler metrics living on the complexified tangent bundle, rather than genuine Kähler metrics.

\subsection{Kähler metrics for Lorentzian metrics of Petrov type $D$}

By the Goldberg-Sachs Theorem [19] (see also [28, Chapter 5]), a Lorentzian metric of Petrov type $D$ admits two null geodesic vector fields which are both shear-free. Even when these satisfy the conditions of Theorem 1, neither one may be gradient, or even near-gradient in the sense of Definition 4.1(ii). Thus they do not give rise to an admissible manifold as in Definition 4.1. However, if one takes $\boldsymbol{k}_{+}$to be one of these geodesic fields, 
and $\boldsymbol{k}_{\text {- }}$ a pre-geodesic field which is a function multiple of the second, it is still possible to form very similar Kähler metrics, as the following proposition shows:

Proposition 10.1. Let $g$ be a Petrov type D metric on an oriented 4-manifold, with null shear-free vector fields $\boldsymbol{k}_{ \pm}$, with $\boldsymbol{k}_{+}$geodesic, $\boldsymbol{k}_{-}$pre-geodesic and $g\left(\boldsymbol{k}_{+}, \boldsymbol{k}_{-}\right)<0$. Assume also that $J:=J_{g, \boldsymbol{k}_{ \pm}}$is an admissible complex structure. Set $p:=1 / \sqrt{-g\left(\boldsymbol{k}_{+}, \boldsymbol{k}_{-}\right)}$. Suppose $u$ is a smooth function on $M$ and $f$ a smooth positive function defined on the range of $u$, for which $\nabla(f(u) / p) \in \Gamma(\mathcal{V})$ for $\mathcal{V}=\operatorname{span}\left(\boldsymbol{k}_{+}, \boldsymbol{k}_{-}\right)$. Then

$$
g_{K}=d\left(f(u) p \boldsymbol{k}_{+}^{b}\right)(\cdot, J \cdot)
$$

is Kähler in any region where $\iota^{\boldsymbol{k}_{+}}<0$ and $d_{\boldsymbol{k}_{+}}(f(u) p)<0$.

Proof. Setting $\tilde{\boldsymbol{k}}_{ \pm}:=p \boldsymbol{k}_{ \pm}$, note that $g\left(\tilde{\boldsymbol{k}}_{+}, \tilde{\boldsymbol{k}}_{-}\right)=-1$ and $\nabla_{\tilde{\boldsymbol{k}}_{+}} \tilde{\boldsymbol{k}}_{+}=\left(d_{\boldsymbol{k}_{+}} p\right) \tilde{\boldsymbol{k}}_{+}$, so that $\tilde{\boldsymbol{k}}_{+}$is null pre-geodesic. Observe also that $J_{g, \tilde{\boldsymbol{k}}_{ \pm}}=J_{g, \boldsymbol{k}_{ \pm}}$. Now consider $\omega:=d\left(f(u) \tilde{\boldsymbol{k}}_{+}^{b}\right)$. For the usual orthonormal frame $\boldsymbol{x}, \boldsymbol{y}$ on $\mathcal{H}$, it follows from a calculation as in Theorem 3 that $\omega\left(\tilde{\boldsymbol{k}}_{+}, \boldsymbol{x}\right)=\omega\left(\tilde{\boldsymbol{k}}_{+}, \boldsymbol{y}\right)=0$, and similarly $\omega(\boldsymbol{x}, \boldsymbol{y})=-f p \iota^{\boldsymbol{k}_{+}}$, and

$$
\omega\left(\tilde{\boldsymbol{k}}_{+}, \tilde{\boldsymbol{k}}_{-}\right)=-\left(f^{\prime} d u\left(\tilde{\boldsymbol{k}}_{+}\right)+f d_{\boldsymbol{k}_{+}} p\right) .
$$

Thus $\omega$ will be symplectic if $f\left(f^{\prime} d u\left(\tilde{\boldsymbol{k}}_{+}\right)+f d_{\boldsymbol{k}_{+}} p\right) \iota^{\boldsymbol{k}_{+}} \neq 0$.

For $J$-invariance of $\omega$, again the crucial test is when one vector field lies in $\mathcal{V}$ and the other in $\mathcal{H}$. Since we know $\omega$ vanishes on the null pre-geodesic vector field $\tilde{\boldsymbol{k}}_{+}$and a vector field in $\mathcal{H}$, we need to show the same on $\tilde{\boldsymbol{k}}_{-}$and such a vector field. As in Theorem 3, we calculate, noting that $g\left(\left[\boldsymbol{k}_{-}, \boldsymbol{x}\right], \boldsymbol{k}_{+}\right)=0$ as usual, and relying on the constancy of $g\left(\tilde{\boldsymbol{k}}_{+}, \tilde{\boldsymbol{k}}_{-}\right)$:

$$
\begin{aligned}
\omega\left(\tilde{\boldsymbol{k}}_{-}, \boldsymbol{x}\right) & =-f^{\prime} d u(\boldsymbol{x}) g\left(\tilde{\boldsymbol{k}}_{+}, \tilde{\boldsymbol{k}}_{-}\right)+f\left(-g\left(\left[\tilde{\boldsymbol{k}}_{-}, \boldsymbol{x}\right], \tilde{\boldsymbol{k}}_{+}\right)\right) \\
& =f^{\prime} d u(\boldsymbol{x})+f p\left(d_{\boldsymbol{x}} p\right) g\left(\boldsymbol{k}_{-}, \boldsymbol{k}_{+}\right)=f^{\prime} d_{\boldsymbol{x}} u-f\left(d_{\boldsymbol{x}} p\right) / p .
\end{aligned}
$$

Vanishing of this follows if $d_{\boldsymbol{x}}(\log f(u))=d_{\boldsymbol{x}}(\log p)$. Vanishing for any $\boldsymbol{x}$ with values in $\mathcal{H}$ will thus hold if $\log (f(u) / p)$, or equivalently $f(u) / p$, has a vertical gradient.

Tameness of $J$ in the region specified by the inequalities in the theorem follows from the above calculations of $\omega(\boldsymbol{x}, \boldsymbol{y})$ (note that $f, p$ are positive) and $\omega\left(\tilde{\boldsymbol{\kappa}}_{+}, \tilde{\boldsymbol{k}}_{-}\right)$, where for the latter we note that $-\left(f^{\prime} d u\left(\tilde{\boldsymbol{k}}_{+}\right)+\right.$ $\left.f d_{\boldsymbol{k}_{+}} p\right)=-\left(f^{\prime} p d u\left(\boldsymbol{k}_{+}\right)+f d_{\boldsymbol{k}_{+}} p\right)=-d_{\boldsymbol{k}_{+}}(f(u) p)$ must be positive.

Note that $\tilde{\boldsymbol{k}}_{ \pm}$satisfy the conditions of Theorem 2, rather than Theorem 1 .

Remark 10.2. Remark 3.3 applies here, while Remark 4.3 follows analogously, so that $g_{K}$ of Proposition 10.1 is ambihermitian.

\subsection{A Kähler metric on Kerr spacetime}

The Kerr spacetime can be partitioned in the form $M=M^{\prime} \cup \Sigma \cup M^{\prime \prime}$, where $M^{\prime}, M^{\prime \prime}$ are open submanifolds, and $\Sigma$ is a totally geodesic hypersurface called the equatorial plane, which contains a cylinder called the ring singularity, where the Kerr metric $g$ is singular. We construct a Kähler metric $g_{K}$ on a subset of $M^{\prime}$, in the case where the Kerr spacetime is rapidly rotating, a status determined by an inequality between the two constant parameters in $g$. A similar Kähler metric can be constructed on a subset of $M^{\prime \prime}$ if the complex structure we choose is changed by a minus sign on a distribution denoted $\mathcal{H}$ and described below.

The Kerr metric $g$ is initially defined in an open subset of $M:=\mathbb{R}^{2} \times \mathbb{S}^{2}$. In coordinates $\{t, r, \vartheta, \varphi\}$, with $0<\vartheta<\pi$ and $0 \leq \varphi \leq 2 \pi, g$ has components

$$
\begin{gathered}
g_{t t}=-1+\frac{2 m r}{\rho^{2}}, \quad g_{r r}=\frac{\rho^{2}}{\Delta}, \quad g_{9 \vartheta}=\rho^{2}, \\
g_{\varphi \varphi}=\left[r^{2}+a^{2}+\frac{2 m r a^{2} \sin ^{2} \vartheta}{\rho^{2}}\right] \sin ^{2} \vartheta \quad, \quad g_{\varphi t}=g_{t \varphi}=-\frac{2 m r a \sin ^{2} \vartheta}{\rho^{2}},
\end{gathered}
$$


all other components being zero, with $a, m$ positive parameters and

$$
\rho^{2}:=r^{2}+a^{2} \cos ^{2} \vartheta, \quad \Delta:=r^{2}-2 m r+a^{2} .
$$

Kerr spacetime is designated as rapidly rotating if $a>m$, and in that case $\Delta$ has no real roots. Our choice of the rapidly rotating version is made for convenience only, to simplify the singular region of the metric.

As a (Ricci-flat) Petrov Type $D$ metric, $\boldsymbol{g}$ ’s two geodesic and shear-free null vector fields $\boldsymbol{k}_{ \pm}$are everywhere linearly independent and given by

$$
\boldsymbol{k}_{ \pm}:= \pm \partial_{r}+\frac{r^{2}+a^{2}}{\Delta} \partial_{t}+\frac{a}{\Delta} \partial_{\varphi}
$$

(see [28, p. 79ff.]).

We consider as usual the almost complex structure $J:=J_{g, \boldsymbol{k}_{ \pm}}$. The orientation is fixed by choosing on the spacelike distribution $\mathcal{H}=\operatorname{span}\left(\boldsymbol{k}_{+}, \boldsymbol{k}_{-}\right)^{\perp}$, the orthonormal pair

$$
E_{2}:=\frac{1}{\rho} \partial_{\vartheta}, \quad E_{3}:=\frac{1}{\rho \sin \vartheta} \partial_{\varphi}+\frac{a \sin \vartheta}{\rho} \partial_{t}
$$

and taking $J E_{2}:=E_{3}, J E_{3}:=-E_{2}$. (The notations $E_{2}, E_{3}$ conform with [28]).)

To construct Kähler metrics, we will apply Proposition 10.1. First, we verify integrability of $J$, by checking the conditions of Theorem 1. Now $\boldsymbol{k}_{ \pm}$are shear-free, and conditions (i) of Theorem 1 hold because

$$
\left[\boldsymbol{k}_{ \pm}, E_{2}\right]=\mp \frac{r}{\rho^{2}} E_{2}, \quad\left[\boldsymbol{k}_{ \pm}, E_{3}\right]=\mp \frac{r}{\rho^{2}} E_{3},
$$

as can be easily verified using $\left[28\right.$, p. 95-6]. ${ }^{2}$

Next, one easily calculates that $g\left(\boldsymbol{k}_{+}, \boldsymbol{k}_{-}\right)=-2 \rho^{2} / \Delta<0$, so that

$$
p:=\sqrt{\frac{\Delta}{2}} \frac{1}{\rho} .
$$

Note that $p$ is a function of only $r$ and $\vartheta$. Let $u=e^{h(r)} p$, with $h$ to be determined later, and $f(u)=u$, which is positive on the (positive) range of $u$. The conditions of Proposition 10.1 we want satisfied can be written in the form

$$
\begin{aligned}
& {[\nabla \log (f(u))]^{\mathcal{H}}=[\nabla \log p]^{\mathcal{H}},} \\
& d_{\boldsymbol{k}_{+}}[\log (f(u) p)]<0 .
\end{aligned}
$$

Now the inverse matrix to that of $g$ has the nonzero components at the same entries as those of $g$, and $g^{r r}=$ $1 / \rho^{2}>0$. Thus $\nabla h(r)=g^{r r} h_{r} \partial_{r}$, which lies in $V$. Thus the first condition in (47) is automatically satisfied, since $\log f(u)=\log p+h(r)$. The second condition is just

$$
\begin{aligned}
d_{\boldsymbol{k}_{+}}(h)=h_{r} & <-2 d_{\boldsymbol{k}_{+}} \log p=-\left(\log \left(p^{2}\right)\right)_{r} \\
& =\left(\log \rho^{2}\right)_{r}-(\log \Delta)_{r}=\left(2 r / \rho^{2}-2(r-m) / \Delta\right) .
\end{aligned}
$$

As $1 / \rho^{2}>1 /\left(r^{2}+a^{2}+1\right)$, this will be satisfied at any point of $M-\Sigma$ for which $r>0$ if $h(r)=h_{a, m}(r)$ is given by

$$
h(r)=\log \left(\frac{r^{2}+a^{2}+1}{\Delta}\right) .
$$

The function $\iota$, which is, up to sign, the twist function of $\boldsymbol{k}_{+}$, is given (cf. [28]) in the ordered basis $E_{2}, E_{3}$, by

$$
\iota=\frac{2 a \cos \vartheta}{\rho^{2}}
$$

2 Note that on $p$. 95 of [28], there are two occurrences of " $r / \sqrt{\varepsilon \Delta}\left(E_{0} \pm E_{1}\right)$ " which should in fact be " $\rho / \sqrt{\varepsilon \Delta}\left(E_{0} \pm E_{1}\right)$," with $\rho$ given by (43). Also, on p. 96 of [28] the Lie bracket $\left[E_{2}, E_{3}\right]$ should equal " $-r \sqrt{\varepsilon \Delta} \rho^{-3} E_{3}$," not " $-r \sqrt{\varepsilon \Delta} \rho E_{3}$." 
Thus । vanishes only on the totally geodesic hypersurface $\Sigma$ given by $\vartheta=\pi / 2$, known as the "equatorial plane" (a plane is gotten by also fixing a value of $t$ ). Hence $\iota$ will be negative "below" it, so that

$$
\left.g_{\kappa}=d\left(e^{h(r)} p^{2} \boldsymbol{k}_{+}^{b}\right)\right)\left(\cdot, J_{g, \boldsymbol{k}_{ \pm}} \cdot\right)=d\left(\frac{r^{2}+a^{2}+1}{2 \rho^{2}} \boldsymbol{k}_{+}^{b}\right)\left(\cdot, J_{g, \boldsymbol{k}_{ \pm}} \cdot\right)
$$

is a Kähler metric on the set

$$
M^{\prime} \cap\{r>0\}, \text { where } M^{\prime}:=\left\{(t, r, \vartheta, \varphi) \in \mathbb{R}^{2} \times \mathbb{S}^{2} \mid \pi / 2<\vartheta<\pi\right\} .
$$

Note that Dixon describes in [15] a Kähler metric which is obtained by a type of Wick rotation of the Kerr metric, also not defined on the whole spacetime, and whose Kähler form was given earlier in [4]. He also showed this metric is in fact ambitoric, and studied its domain and asymptotic behaviour near the singular sets, including the non-rapidly rotating case.

\subsection{A Kähler metric on NUT spacetime}

One of 3 NUT spacetimes (Newman-Unti-Tamburino) is given in local coordinates $\{u, r, x, y\}$ by

$$
\begin{gathered}
g_{u u}=-|\rho|^{2}\left(r^{2}-2 m r-l^{2}\right), \quad g_{u r}=-1, \quad g_{r y}=2 l \cos x, \\
g_{u y}=2|\rho|^{2} l \cos x\left(r^{2}-2 m r-l^{2}\right), \quad g_{x x}=r^{2}+l^{2}, \\
g_{y y}=-|\rho|^{2}\left(r^{2}-2 m r-l^{2}\right)\left(4 l^{2} \cos ^{2} x\right)+\left(r^{2}+l^{2}\right) \sin ^{2} x,
\end{gathered}
$$

all other components being zero, with $\rho:=-1 /(r+i l)$ and $l$ a positive constant (see [21, 22]).

NUT spacetime is Ricci-flat and topologically an open subset of $\mathbb{R}^{2} \times \mathbb{S}^{2}$. Its two shear-free null vector fields are

$$
\boldsymbol{k}_{+}:=\partial_{r}, \quad \boldsymbol{k}_{-}:=\partial_{u}-\frac{1}{2}|\rho|^{2}\left(r^{2}-2 m r-l^{2}\right) \partial_{r} .
$$

We report without proof that the conditions of Proposition 10.1 hold (with $p=1$ ) so that $g_{K}=d\left(e^{-r} \boldsymbol{k}_{+}^{b}\right)(\cdot, J \cdot)$ will be a Kähler metric on the entire domain of NUT spacetime lying in $S^{2} \times \mathbb{R}^{2}$ (in contrast to the Kerr case).

\subsection{A conformally Kerr metric and its induced Kähler metric}

Our final Petrov type $D$ example is admissible. It is conformal to Kerr spacetime, namely

$$
\tilde{g}:=\frac{\Delta}{\rho^{2}} g,
$$

where $g$ denotes the Kerr metric (42) and the notation is as in (43). The associated almost complex structure $\tilde{J}:=J_{\tilde{g}, \boldsymbol{k}, \tilde{\nabla} r}$ will be defined with respect to $\left\{\boldsymbol{k}_{+}, \widetilde{\nabla} r\right\}$ instead of $\left\{\boldsymbol{k}_{+}, \boldsymbol{k}_{-}\right\}$. With this conformal factor, the gradient $\widetilde{\nabla} r=\partial_{r}$. Definition 4.1's conditions may be verified to yield admissibility. An assoicated metric of the form $g_{K}=d\left(f(r) \boldsymbol{k}_{+}^{b}\right)(\cdot, \tilde{J} \cdot)$ is Kähler on the region $M^{\prime} \backslash\{r=0\}$, where $M^{\prime}$ is given in (49), if $f(r)=e^{-h(r)}$, for $h(r):=\int_{r_{0}}^{r} q(x) d x$ with $r_{0}$ a constant and $q(r)=2(r-m) /\left(r^{2}-2 m r+a^{2}\right)-2 / r$, as can be verified via Theorem 3.

\section{A Lie group example with vector fields which are not shear-free}

Consider the 4-dimensional solvable real Lie algebra $\mathfrak{g}$ with ordered basis $\boldsymbol{k}, \boldsymbol{t}, \boldsymbol{x}, \boldsymbol{y}$ defined by the following Lie bracket relations, where we list only the non-zero ones (up to permuting entries):

$$
[k, x]=y, \quad[t, y]=y, \quad[t, k]=k, \quad[x, y]=y+r k,
$$


with $r$ a nonzero real constant. Four dimensional solvable Lie algebras have been classified using symbolic software, cf. [12] (note that we are choosing a slightly different form for the bracket relations, isomorphic to one of the canonical normal forms given there).

By Lie's third fundamental theorem, there exists a Lie group $\widehat{G}$ whose Lie algebra is $\mathfrak{g}$, and one can take it to be simply connected, as we do. The left invariant vector fields of $\widehat{G}$ give a realization of this Lie algebra, and will be denoted by the same letters.

Define a Lorentzian metric $g$ on $\widehat{G}$ by choosing an inner product on $\mathfrak{g}$ making the above four vectors orthonormal, with $g(\boldsymbol{k}, \boldsymbol{k})=-g(\boldsymbol{t}, \boldsymbol{t})=1$, and then extending it to the tangent bundle of $\widehat{G}$ as a left-invariant metric. Then one easily checks that

$$
g([\boldsymbol{k}, \mathcal{H}], \boldsymbol{k})=g([\boldsymbol{t}, \mathcal{H}], \boldsymbol{t})=g([\boldsymbol{k}, \mathcal{H}], \boldsymbol{t})=g([\boldsymbol{t}, \mathcal{H}], \boldsymbol{k})=0,
$$

where $\mathcal{H}=\operatorname{span}(\boldsymbol{x}, \boldsymbol{y})$. Similarly, using (3), one sees that

$$
\sigma_{1}^{\boldsymbol{k}}=-\sigma_{2}^{\boldsymbol{t}}=0, \quad \sigma_{2}^{\boldsymbol{k}}=\sigma_{1}^{\boldsymbol{t}}=-1 \neq 0,
$$

so that the shear operators of $\boldsymbol{k}$ and $\boldsymbol{t}$ are nonzero and $J \nabla^{o} \boldsymbol{k}=\nabla^{o} \boldsymbol{t}$, where $J=J_{g, \boldsymbol{k}, \boldsymbol{t}}$ is the corresponding admissible almost complex structure (with orientation compatible with the choice $\boldsymbol{y}:=J \boldsymbol{x}$ ). As the conditions of Theorem 1 hold, $J$ is integrable. Next, the twist function of $\boldsymbol{k}$ is $|\iota|=|g(\boldsymbol{k},[\boldsymbol{x}, \boldsymbol{y}])|=|r| \neq 0$, so that the sign of $\boldsymbol{l}$ is fixed by the choice of $r$. As $\boldsymbol{t}$ is a left invariant vector field, $\nabla_{\boldsymbol{t}} \boldsymbol{t}=-\operatorname{ad}_{\boldsymbol{t}}^{*}(\boldsymbol{t})$, (see [11, Proposition 3.18]), where $\operatorname{ad}_{t}^{\star}$ denotes the metric adjoint of the differential at $t$ of the adjoint representation. As this differential is given by the Lie bracket with $\boldsymbol{t}$, it follows from (52) that $g(\boldsymbol{t},[\boldsymbol{t}, \cdot])$ vanishes on the left invariant orthonormal frame $\boldsymbol{k}, \boldsymbol{x}, \boldsymbol{y}, \boldsymbol{t}$ of $\widehat{G}$, so that $\nabla_{\boldsymbol{t}} \boldsymbol{t}=0$. By (52), the orthogonal complement of $\boldsymbol{t}$ is integrable. As $\boldsymbol{t}$ has constant length and is geodesic, one can easily see that $\boldsymbol{t}^{b}$ is closed, hence $\boldsymbol{t}$ is locally gradient, and as $\widehat{G}$ is simply connected, in fact globally a gradient, i.e. $\boldsymbol{t}=\nabla \tau$ for a function $\tau$ on $\widehat{G}$. Given that, and the fact that $g(\boldsymbol{k}, \boldsymbol{k})$ and $g(\boldsymbol{k}, \boldsymbol{t})$ are constant, Theorem 3 yields induced Kähler metrics of the form (19), with Kähler form (18), at least one of which can be defined on all of $\widehat{G}$. In fact, the domain is given by $f \iota<0$ and $f^{\prime} G / \ell-f\left(g\left(\nabla_{\boldsymbol{k}} \boldsymbol{k}, \boldsymbol{t}\right)-d_{\boldsymbol{t}}(g(\boldsymbol{k}, \boldsymbol{k})) / 2\right)=$ $-f^{\prime}-f<0$, so choosing $f(\tau)=e^{\tau}$ and $r<0$ suffices.

We note that while $g$ is admissible, $\boldsymbol{k}$ is not a geometrically distinguished field in this case.

\section{References}

[1] A. B. Aazami, Symplectic 4-manifolds via Lorentzian geometry, Proceedings of the American Mathematical Society, 145 (2017), pp. 387-394.

[2] A. B. Aazami and G. Maschler, Canonical Kähler metrics on classes of Lorentzian 4-manifolds, arXiv:1811.08999.

[3] D. Alekseevsky and F. Zuddas, Cohomogeneity one Kähler and Kähler-Einstein manifolds with one singular orbit l, Annals of Global Analysis and Geometry, 52 (2017), pp. 99--128.

[4] A. N. Aliev and C. Saçlıoğlu, Self-dual fields harbored by a Kerr-Taub-BOLT instanton, Physics Letters B, 632 (2006), pp. 725727.

[5] V. Apostolov, D. M. J. Calderbank and P. Gauduchon, Ambitoric geometry I: Einstein metrics and extremal ambikähler structures, J. Reine Angew. Math. 721 (2016), 109-147.

[6] V. Apostolov, Generalized Goldberg-Sachs theorems for pseudo-Riemannian four-manifolds, Journal of Geometry and Physics, 27 (1998), pp. 185-198.

[7] J. K. Beem, P. Ehrlich, and K. Easley, Global Lorentzian Geometry, vol. 202 of Pure and Applied Mathematics, Marcel Dekker, Inc., 1996.

[8] D. M. J. Calderbank, Selfdual Einstein metrics and conformal submersions, arXiv math/0001041, (2000).

[9] D. M. J. Calderbank and H. Pedersen, Selfdual spaces with complex structures, Einstein-Weyl geometry and geodesics, in Annales de l'institut Fourier, vol. 50, Chartres: L'Institut, 1950-, 2000, pp. 921-964.

[10] A. M. Candela and M. Sánchez, Geodesics in semi-Riemannian manifolds: geometric properties and variational tools, Recent developments in pseudo-Riemannian geometry, (2008), pp. 359-418.

[11] J. Cheeger and D. G. Ebin, Comparison theorems in Riemannian geometry, vol. 9, North-Holland Publishing Company Amsterdam, 1975.

[12] W. A. de Graaf, Classification of solvable Lie algebras, Experimental mathematics, 14 (2005), pp. 15-25.

[13] A. Derdzinski and G. Maschler, Local classification of conformally-Einstein Kähler metrics in higher dimensions, Proceedings of the London Mathematical Society, 87 (2003), pp. 779-819. 
[14] _ Special Kähler-Ricci potentials on compact Kähler manifolds, Journal für die reine und angewandte Mathematik, 593 (2006), pp. 73-116.

[15] K. Dixon, Regular ambitoric 4-manifolds: from Riemannian Kerr to a complete classification, arXiv:1604.03156, (2016).

[16] E. J. Flaherty, Hermitian and Kählerian geometry in relativity, vol. 46 of Lecture Notes in Physics, Springer, 1976.

[17] J. L. Flores and M. Sánchez, On the geometry of pp-wave type spacetimes, in Analytical and Numerical Approaches to Mathematical Relativity, Springer, 2006, pp. 79-98.

[18] P. Gauduchon, Variétés Lorentziennes de type hermitien à tenseur de Ricci nul, preprint, (1993).

[19] J. Goldberg and R. Sachs, Republication of: A theorem on Petrov types, General Relativity and Gravitation, 41 (2009), pp. 433444.

[20] A. Harris and G. Paternain, Conformal great circle flows on the 3-sphere, Proceedings of the American Mathematical Society, (2015).

[21] W. M. Kinnersley, Type D gravitational fields, PhD thesis, California Institute of Technology, 1969.

[22] - Type D vacuum metrics, Journal of Mathematical Physics, 10 (1969), pp. 1195-1203.

[23] T. Leistner and D. Schliebner, Completeness of compact Lorentzian manifolds with abelian holonomy, Mathematische Annalen, 364 (2016), pp. 1469-1503.

[24] G. Maschler, Central Kähler metrics, Transactions of the American Mathematical Society, 355 (2003), pp. $2161--2182$.

[25] P. Nurowski, Einstein equations and Cauchy-Riemann geometry, PhD thesis, SISSA, 1993.

[26] P. Nurowski and A. Trautman, Robinson manifolds as the Lorentzian analogs of Hermite manifolds, Differential Geometry and its Applications, 17 (2002), pp. 175-195.

[27] B. O'Neill, Semi-Riemannian geometry. With applications to relativity, vol. 103 of Pure and Applied Mathematics, Academic Press, 1983.

[28] - The geometry of Kerr black holes, Wellesley, Mass.: AK Peters, 1995.

[29] R. Sachs, Gravitational waves in general relativity. vi. the outgoing radiation condition, in Proceedings of the Royal Society of London A: Mathematical, Physical and Engineering Sciences, vol. 264, The Royal Society, 1961, pp. 309-338.

[30] I. P. Silva, J. L. Flores, and J. Herrera, Rigidity of geodesic completeness in the Brinkmann class of gravitational wave spacetimes, arXiv:1605.03619, (2016).

[31] W. P. Thurston and J. W. Milnor, The geometry and topology of three-manifolds, Princeton University Princeton, 1979.

[32] A. Trautman, On complex structures in physics, in On Einstein's path, Springer, 1999, pp. 487-501.

[33] D. Varolin, Three variations on a theme in complex analytic geometry, Analytic and Algebraic Geometry, IAS/Park City Math. Ser., 17 (2008), pp. 183-294.

[34] S.-T. Yau, Open problems in geometry, in Proceedings of Symposia in Pure Mathematics, vol. 54, 1993, pp. 1-28. 